\title{
CHEMICAL EVOLUTION OF PROTOPLANETARY DISKS—THE EFFECTS OF VISCOUS ACCRETION, TURBULENT MIXING, AND DISK WINDS
}

\author{
D. Heinzeller ${ }^{1,3}$, H. Nomura ${ }^{1}$, C. Walsh ${ }^{2}$, and T. J. Millar ${ }^{2}$ \\ ${ }^{1}$ Department of Astronomy, Graduate School of Science, Kyoto University, Kyoto 606-8502, Japan; dominikus@ kusastro.kyoto-u.ac.jp \\ ${ }^{2}$ Astrophysics Research Centre, School of Mathematics and Physics, Queen's University Belfast, Belfast, BT7 1NN, UK \\ Received 2010 September 18; accepted 2011 February 16; published 2011 March 31
}

\begin{abstract}
We calculate the chemical evolution of protoplanetary disks considering radial viscous accretion, vertical turbulent mixing, and vertical disk winds. We study the effects on the disk chemical structure when different models for the formation of molecular hydrogen on dust grains are adopted. Our gas-phase chemistry is extracted from the UMIST Database for Astrochemistry (Rate06) to which we have added detailed gas-grain interactions. We use our chemical model results to generate synthetic near- and mid-infrared local thermodynamic equilibrium line emission spectra and compare these with recent Spitzer observations. Our results show that if $\mathrm{H}_{2}$ formation on warm grains is taken into consideration, the $\mathrm{H}_{2} \mathrm{O}$ and $\mathrm{OH}$ abundances in the disk surface increase significantly. We find that the radial accretion flow strongly influences the molecular abundances, with those in the cold midplane layers particularly affected. On the other hand, we show that diffusive turbulent mixing affects the disk chemistry in the warm molecular layers, influencing the line emission from the disk and subsequently improving agreement with observations. We find that $\mathrm{NH}_{3}, \mathrm{CH}_{3} \mathrm{OH}, \mathrm{C}_{2} \mathrm{H}_{2}$, and sulfur-containing species are greatly enhanced by the inclusion of turbulent mixing. We demonstrate that disk winds potentially affect the disk chemistry and the resulting molecular line emission in a manner similar to that found when mixing is included.
\end{abstract}

Key words: accretion, accretion disks - astrochemistry - infrared: planetary systems - planetary systems protoplanetary disks - turbulence

Online-only material: color figures

\section{INTRODUCTION}

Protoplanetary disks are a natural and active environment for the creation of simple and complex molecules. Understanding their physical and chemical evolution is key to a deeper insight into the processes of star and planetary system formation and corresponding chemical evolution. Protoplanetary disks are found encompassing young stars in star-forming regions and typically disperse on timescales of less than a few million years (e.g., Haisch et al. 2001, 2006; Watson et al. 2007). Observations of thermal dust emission from these systems date back to the 1980s (see, e.g., Lada \& Wilking 1984; Beckwith et al. 1990) and gave important insight into the structure and evolution of disks (see, e.g., Natta et al. 2007 for a review), however, inferring the properties of protoplanetary disks from dust continuum observations has several shortcomings: the dust component is only $\approx 1 \%$ of the total mass contained in the disk and its properties are expected to change with dust growth and planet formation. Also, dust spectral features are too broad to study the disk kinematics (see, e.g., Carmona 2010), and observations suggest that the gas and dust temperatures can differ considerably, particularly in the UV- and X-ray-heated surface layers of the inner disk (e.g., Qi et al. 2006; Carmona et al. 2007).

The observation of molecular line emission from disks at (sub)millimeter wavelengths is limited by the low spatial resolution and sensitivity of existing facilities and target the rotational transitions of abundant molecules in the outer $(\geqslant 50 \mathrm{AU})$ regions of the disk (see, e.g., Dutrey et al. 2007 for a review). Planet formation, however, most likely occurs at much

3 Current address: Meteorological Service of New Zealand Ltd, 30 Salamanca Rd, Kelburn, Wellington 6012, New Zealand. smaller radii (Armitage 2007). The superior sensitivity and spatial and spectral resolution of the Atacama Large Millimeter Array (ALMA), currently under construction, are required to observe the (sub)millimeter emission lines from the inner regions of protoplanetary disks in nearby star-forming regions to enable direct study of the physical and chemical structure of the inner disk.

Near- and mid-infrared observations, on the other hand, can probe the warm planet-forming regions (see, e.g., Najita et al. 2007 for a review). The $4.7 \mu \mathrm{m}$ CO line emission has been detected at radii $<1 \mathrm{AU}$, using, for example, Keck/NIRSPEC, Gemini/Phoenix, and VLT/CRIRES (e.g., Carr 2007; Pontoppidan et al. 2008; Brittain et al. 2009; van der Plas et al. 2009). The Spitzer Space Telescope has been used to spectroscopically study the inner disk at mid-infrared wavelengths. These observations show a rich spectrum of emission lines from simple organic molecules (e.g., Carr \& Najita 2008; Salyk et al. 2008; Pontoppidan et al. 2010) as well as from complex polycyclic aromatic hydrocarbons (PAHs; e.g., Habart et al. 2006; Geers et al. 2006). They suggest high abundances of $\mathrm{H}_{2} \mathrm{O}, \mathrm{HCN}$, and $\mathrm{C}_{2} \mathrm{H}_{2}$ and provide evidence that the disk supports an active chemistry. $\mathrm{C}_{2} \mathrm{H}_{2}$ and $\mathrm{HCN}$ have also been detected in absorption toward IRS 46 and GV Tauri (Lahuis et al. 2006; Gibb et al. 2007). A particularly interesting case is $\mathrm{CO}_{2}$, which has been reported to be abundant in the circumstellar disk of AA Tauri at $\sim 1.2$ AU (Carr \& Najita 2008), in IRS 46 (Lahuis et al. 2006), and in several other sources (e.g., Pontoppidan et al. 2010), but which is not detected in the majority of sources observed by Spitzer. The recently launched Herschel Space Observatory and future space telescopes (e.g., the Space Infrared Telescope for Cosmology and Astrophysics, SPICA, and the James Webb Space Telescope, JWST) will enable the observation of molecular line emission in the mid- to far-infrared with unprecedented 
sensitivity and spectral resolution and will help address these controversial findings.

Due to increasing computational power, the theoretical study of the physical and chemical evolution of protoplanetary disks is entering its Golden Age. As a consequence of the observational limitations in the pre-Spitzer era, many numerical models focused only on the outer disk (e.g., Aikawa et al. 2002; Willacy 2007). With the launch of Spitzer, however, the chemistry of the inner disk has increased in importance (e.g., Woods \& Willacy 2009). Unfortunately, complete models, including the physical and chemical evolution of the gas and the dust in the disk, coupled with a consistent treatment of the radiative transfer, are still beyond reach and several compromises have to be made.

One possibility is to ignore the chemical evolution and assume chemical equilibrium holds throughout the disk to investigate the physical structure in detail (e.g., Kamp \& Dullemond 2004; Jonkheid et al. 2004). Pioneering work by Gorti \& Hollenbach (2004) and Nomura \& Millar (2005) combined a basic disk chemistry with a self-consistent description of the density and temperature structure taking into account the gas thermal balance, the stellar irradiation, and the dust grain properties. Recently, Woitke et al. (2009a) calculated radiation thermochemical models of protoplanetary disks, including frequencydependent two-dimensional dust continuum radiative transfer, kinetic gas-phase and photochemistry, ice formation and nonLTE heating and cooling. These studies show, in accordance with the observations, that the stellar UV and X-ray irradiation increases the gas temperature considerably in the surface layer of the disk, altering the disk chemistry drastically in this region.

Contrary to that described above, several authors instead focused on combining the physical motion of material in the disk with time-dependent chemical network calculations (e.g., Aikawa et al. 1999; Markwick et al. 2002; Ilgner et al. 2004; Nomura et al. 2009). Radial inward motion due to the viscous accretion and vertical motion due to inherent turbulent mixing or disk winds have the potential to change the disk chemistry significantly, depending on the timescales of the transport processes. Molecules, initially frozen out on dust grains, can evaporate and increase the gas-phase abundances, provided that accretion and vertical mixing are comparable to, or faster than, the chemical reactions and the destruction by incident stellar irradiation. First steps toward including the accretion flow in the chemical network have been carried out by Aikawa et al. (1999), Markwick et al. (2002), and Ilgner et al. (2004). Nomura et al. (2009) calculated the effect of accretion along streamlines in steady $(1+1)$-dimensional $\alpha$-disk models for chemical networks containing more than 200 species. They found that the chemical evolution is influenced globally by the inward accretion process, resulting in, e.g., enhanced methanol abundances in the inner disk. Ilgner et al. (2004) also studied vertical mixing using the diffusion approximation and concluded that vertical mixing changes the abundances of the sulfur-bearing species. Their disk model, however, assumed that the gas and dust temperatures were coupled everywhere in the disk and ignored stellar irradiation processes. Semenov et al. (2006) investigated gas-phase CO abundances in a twodimensional disk model, allowing for radial and vertical mixing using a reduced chemical network of 250 species and 1150 chemical reactions, and find the mixing processes strongly affect the CO abundances in the disk. Willacy et al. (2006) studied the effects of vertical diffusive mixing driven by turbulence and found that this can greatly affect the column density of various molecules and increase the size of the intermediate, warm molecular layer in the disk.

In this study, we consider the effects of inward accretion motion and vertical turbulent mixing to investigate to what extent the abundances of molecules such as $\mathrm{H}_{2} \mathrm{O}, \mathrm{CO}_{2}$, and $\mathrm{CH}_{3} \mathrm{OH}$ are affected in the inner disk. As an alternative driving force for vertical transport, we investigate the effect of upward disk winds on the disk chemistry. Several kinds of disk wind models, such as magnetocentrifugally driven winds (e.g., Blandford \& Payne 1982; Shu et al. 1994) and photoevaporation winds (e.g., Shu et al. 1993), have been suggested thus far. Recently, Suzuki \& Inutsuka (2009) studied disk winds driven by MHD turbulence in the inner disk and found that large-scale channel flows develop most effectively at 1.5-2 disk scale heights, i.e., approximately in those layers where molecular line emission is generated. The disk model in our study is from Nomura \& Millar (2005) including X-ray heating as described in Nomura et al. (2007) and accounts for decoupling of the gas and dust temperatures in the disk surface due to stellar irradiation. We use a large chemical network and allow for adsorption and desorption onto and from dust grains. We also calculate mid-IR dust continuum and molecular line emission spectra assuming local thermodynamic equilibrium (LTE) and compare our results to recent observations. In Section 2, we describe our model in detail. In Section 3, we present and discuss our results, before drawing our final conclusions in Section 4.

\section{MODEL}

\subsection{Disk Structure}

Our physical disk model is from Nomura \& Millar (2005) with the addition of X-ray heating as described in Nomura et al. (2007). They compute the physical structure of a steady, axisymmetric protoplanetary disk surrounding a typical T Tauri star with mass $M=0.5 M_{\odot}$, radius $R=2 R_{\odot}$, effective temperature $T_{\text {eff }}=4000 \mathrm{~K}$, and luminosity $L=0.87 L_{\odot}$. We adopt the $\alpha$-disk model (Shakura \& Sunyaev 1973) to obtain the surface density profile from an equation for angular momentum conservation (e.g., Pringle 1981, Equation (3.9)). We assume an accretion rate of $\dot{M}=10^{-8} M_{\odot} \mathrm{yr}^{-1}$ and a viscosity parameter of $\alpha=0.01$.

We denote the radius in the cylindrical coordinate system with $s$ to distinguish it from the radial distance from the central star, i.e., $r=\sqrt{s^{2}+z^{2}}$, where $z$ represents the height from the disk midplane.

The disk is calculated between $s=0.01 \mathrm{AU}$ and $300 \mathrm{AU}$ in a $(1+1)$-dimensional model, which corresponds to a total disk mass of $2.4 \times 10^{-2} M_{\odot}$ for the values of $\alpha$ and $\dot{M}$ given above. The steady gas temperature and density distributions of the disk are obtained self-consistently by iteratively solving the equations for hydrostatic equilibrium in the vertical direction and local thermal balance between heating and cooling of gas. To solve the equation for hydrostatic equilibrium (Nomura et al. 2007, Equation (11)), the condition

$$
\int_{-z_{\infty}}^{+z_{\infty}} \rho_{\mathrm{gas}}(s, z) d z=\Sigma_{\mathrm{gas}}(s)
$$

with $\rho_{\text {gas }}\left(s, z_{\infty}\right)=10^{-18} \mathrm{~g} \mathrm{~cm}^{-3}$ is imposed (Nomura \& Millar 2005). The heating sources of the gas are grain photoelectric heating by FUV photons and X-ray heating by hydrogen ionization, while cooling occurs through gas-grain collisions and by line transitions (for details, see Nomura \& Millar 2005, Appendix A; Nomura et al. 2007). 
The dust temperature is computed assuming radiative equilibrium between the absorption of stellar radiation and resulting thermal emission by the dust grains. Viscous heating is only taken into account in the disk midplane since its effects in the disk surface are comparably small for $\alpha=0.01$ (Glassgold et al. 2004). In the midplane, the density is high and the gas and dust temperatures are tightly coupled. Note that it is often assumed that the gas temperature is coupled with the dust temperature everywhere in the disk. This is not true in the disk surface which is strongly irradiated by the parent star. The dust temperature is calculated using an iterative radiative transfer calculation by means of the short characteristic method in spherical coordinates, with initial dust temperature profiles obtained from the variable Eddington factor method (see Nomura 2002; Dullemond \& Turolla 2000; Dullemond et al. 2002 for details).

The stellar X-ray and UV radiation fields are based on spectral observations of TW Hydrae, a classical T Tauri star. The X-ray spectrum is obtained by fitting a two-temperature thin thermal plasma model to archival XMM-Newton data (mekal model, $L_{\mathrm{X}} \sim 10^{30} \mathrm{erg} \mathrm{s}^{-1}$ for $0.1 \mathrm{keV}, \ldots, 10 \mathrm{keV}$ ) and is slightly softer than that of typical $\mathrm{T}$ Tauri stars with temperatures of about $2 \mathrm{keV}$ in their quiescent phase (cf. Wolk et al. 2005). The stellar UV radiation field has three components: photospheric blackbody radiation, hydrogenic thermal bremsstrahlung radiation, and strong Ly $\alpha$ emission $\left(L_{\mathrm{FUV}} \sim 10^{31} \mathrm{erg} \mathrm{s}^{-1}\right.$ for $6 \mathrm{eV}, \ldots, 13 \mathrm{eV})$. The photochemistry used in this paper is described in detail in Walsh et al. (2010). Note that the scattering of $\mathrm{Ly} \alpha$ emission by atomic hydrogen is not taken into account. On the other hand, the Ly $\alpha$ emission does not affect the molecular abundances significantly when the dust grains are well mixed with the gas (Fogel et al. 2011). We include the interstellar UV radiation field (Draine 1978; van Dishoeck \& Black 1982); however, the interstellar UV and X-ray radiation fields are negligible compared to the central star (for details, see Nomura et al. 2007).

The mass density, gas temperature, and dust temperature for our disk model are displayed in Figure 1, top, middle, and bottom, respectively. A logarithmic spacing of the grid cells is adopted in radial direction, while the vertical structure is calculated on a linear grid. The number of grid cells used in this work is $35 \times 30$ for $0.5 \mathrm{AU} \leqslant s \leqslant 300 \mathrm{AU}$ and $0 \leqslant z / s \leqslant 0.8$. While this resolution is relatively coarse, its consequences for the line emission are limited due to the interpolation method during the line-of-sight integration (cf. Section 2.7).

A more detailed description of the background theory and calculation of the disk physical model, along with further references, is given in Nomura \& Millar (2005), Nomura et al. (2007), and Walsh et al. (2010).

\subsection{Dust Properties}

The dust properties are important as they influence the physical and chemical structure of the disk in several ways. As the dominant source of opacity, they determine the dust temperature and the UV radiation field in the disk. They also affect the gas temperature, because photoelectric heating by UV photons is the dominant heating mechanism toward the disk surface. Further, the total surface area of the dust grains influences the molecular hydrogen abundance through the $\mathrm{H}_{2}$ formation rates on grains and determines the freeze-out rate of chemical species onto the grain mantle throughout the disk (see Section 2.4). Finally, due to their dominant contribution to the opacity, the dust grain model also determines the emerging continuum spectrum.
For consistency, we adopt the same dust grain model for the spectral calculations as for the underlying protoplanetary disk (Nomura \& Millar 2005). We assume the dust grains to be compact and spherical, and to consist of silicate grains, carbonaceous grains and water ice. We further assume that the dust and the gas are well mixed and that the gas-to-dust mass ratio is uniform $\left(\rho_{\text {dust }} / \rho_{\text {gas }}=0.01\right)$ in the disk. The size distribution is taken from Weingartner \& Draine (2001a) to reproduce the observed extinction in dense clouds, with minimum and maximum sizes for the grains of $3.5 \AA$ and $\sim 10 \mu \mathrm{m}$, respectively. The heating of PAHs is included in the model for photoelectric heating (Weingartner \& Draine 2001b). For further details, see also Appendix D in Nomura \& Millar (2005).

\subsection{Gas-phase Reaction Network}

For the calculation of the chemical evolution in the disk, we use a subset of the UMIST Database for Astrochemistry, or Rate06 (Woodall et al. 2007), neglecting only those species, and thus reactions, containing fluorine and phosphorus (Walsh et al. 2010). Since we are primarily interested in those regions where molecular line emission originates and where most molecules are in the gas phase, we neglect grain-surface reactions excepting $\mathrm{H}_{2}$ grain formation (see Section 2.4). In summary, our gas-phase reaction network contains 375 atomic, molecular, and ionic species, ranging from atomic hydrogen $\mathrm{H}$ to relatively complex molecules such as $\mathrm{H}_{3} \mathrm{C}_{9} \mathrm{~N}^{+}$with mass $m=125 \mu \mathrm{m}$ (see Table 1 for a list of species of particular interest in this study and their binding energies on dust grains). The reaction network consists of 4346 reactions including 3957 two-body reactions, 214 photochemical reactions, 164 X-ray/ cosmic-ray-induced photoreactions, and 11 reactions for direct cosmic-ray ionization.

\subsection{Gas-Grain Interactions}

The formation of molecular hydrogen on the surface of dust grains is thought to be the most efficient means of forming $\mathrm{H}_{2}$ directly from atomic hydrogen. Except for this process, we neglect grain-surface reactions of icy mantle species, but allow for adsorption onto and desorption from dust grains. Hence, except for atomic hydrogen, a particle that hits a dust grain can either detach without change or freeze out and remain in the icy grain mantle from where it may eventually evaporate. Atomic hydrogen forms $\mathrm{H}_{2}$, freezes out onto the grain mantle, or evaporates without reaction.

For the $\mathrm{H}_{2}$ grain formation rates, we consider two different models. In model $\mathrm{A}$, we refer to early work by Gould \& Salpeter (1963) and Hollenbach \& Salpeter (1971), who derive a grain formation rate for imperfectly shaped grain surfaces of

$$
\begin{aligned}
\dot{n}\left(\mathrm{H}_{2}\right)_{\mathrm{A}}^{\mathrm{gr}} & =\frac{1}{2} n_{\mathrm{H}} \cdot v_{\mathrm{H}}^{\text {th }}\left(T_{\mathrm{gas}}\right) n_{\mathrm{dust}} \pi\left\langle a^{2}\right\rangle \alpha_{\mathrm{H}} \\
& =n_{\mathrm{H}} \cdot 2 \times 10^{-18} \mathrm{~s}^{-1} n_{\mathrm{tot}}\left(\frac{T_{\mathrm{gas}}}{300 \mathrm{~K}}\right)^{1 / 2} .
\end{aligned}
$$

In Equation (2), $v_{\mathrm{H}}^{\mathrm{th}}\left(T_{\text {gas }}\right)$ is the thermal relative velocity of hydrogen atoms,

$$
v_{\mathrm{H}}^{\mathrm{th}}\left(T_{\mathrm{gas}}\right)=\left(\frac{8 k_{\mathrm{B}} T_{\mathrm{gas}}}{\pi m_{\mathrm{H}}}\right)^{1 / 2},
$$




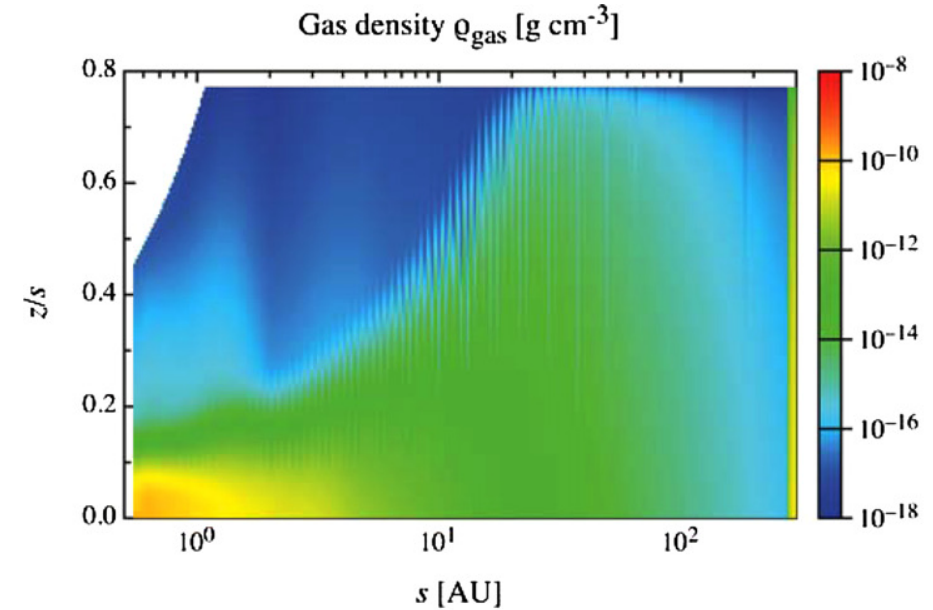

Gas temperature $T_{\text {gas }}[\mathrm{K}]$

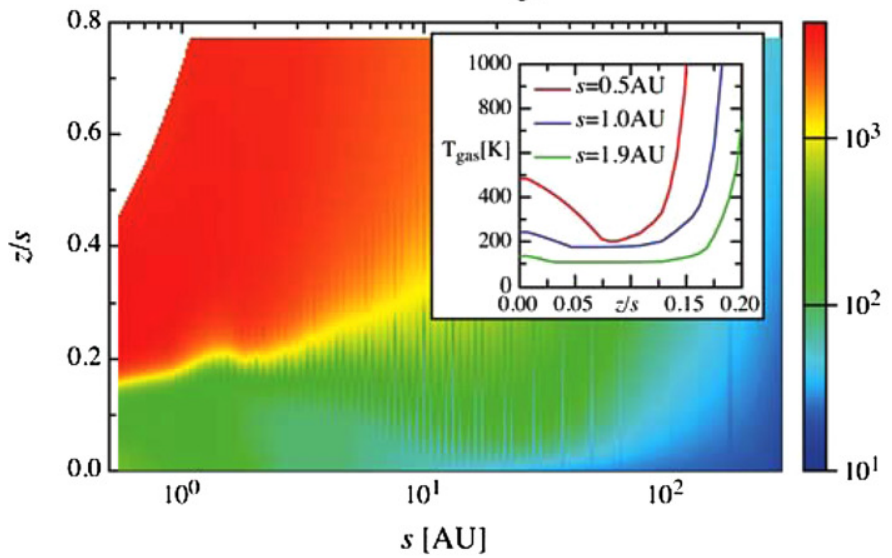

Dust temperature $T_{\text {dust }}[\mathrm{K}]$

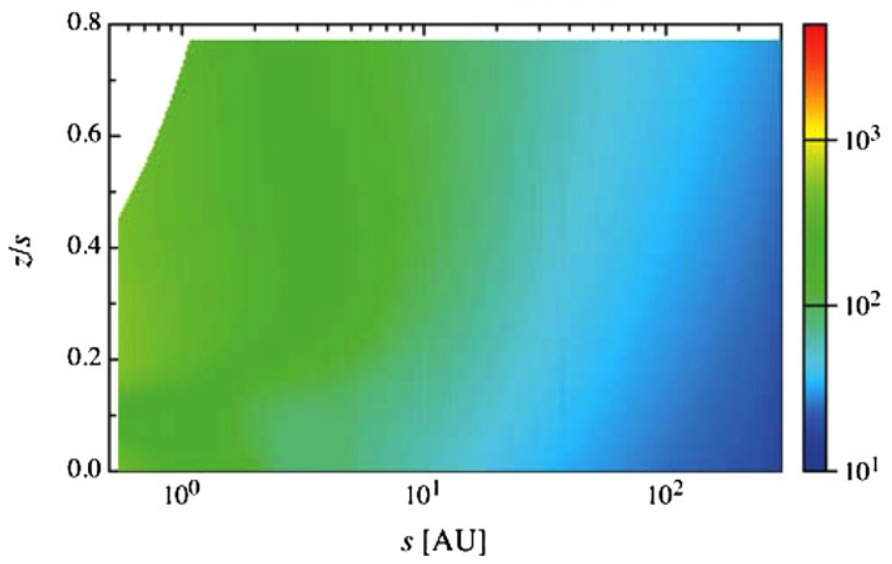

Gas density along streamlines $z / h=$ const.

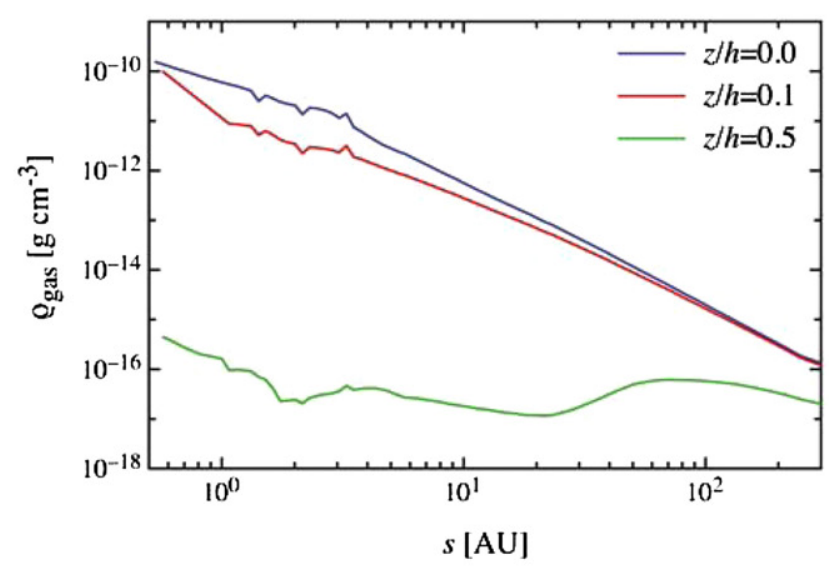

Gas temperature along streamlines $z / h=$ const.

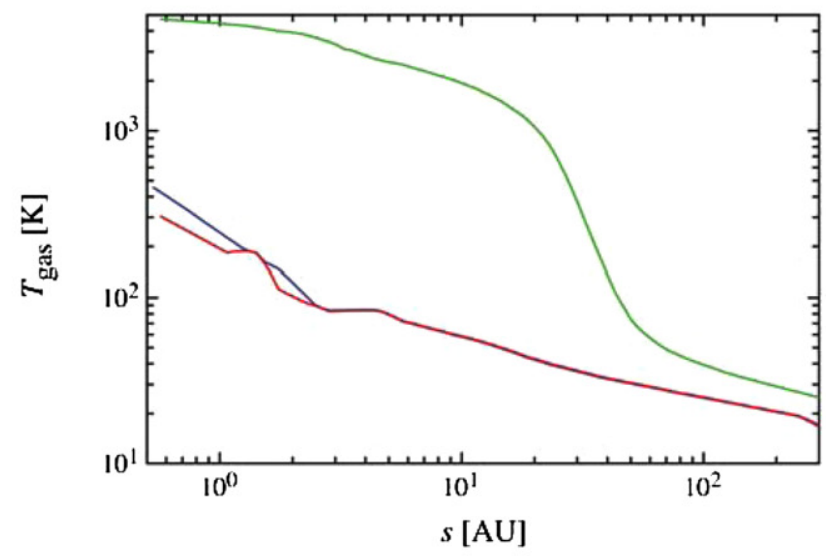

Dust temperature along streamlines $z / h=$ const.

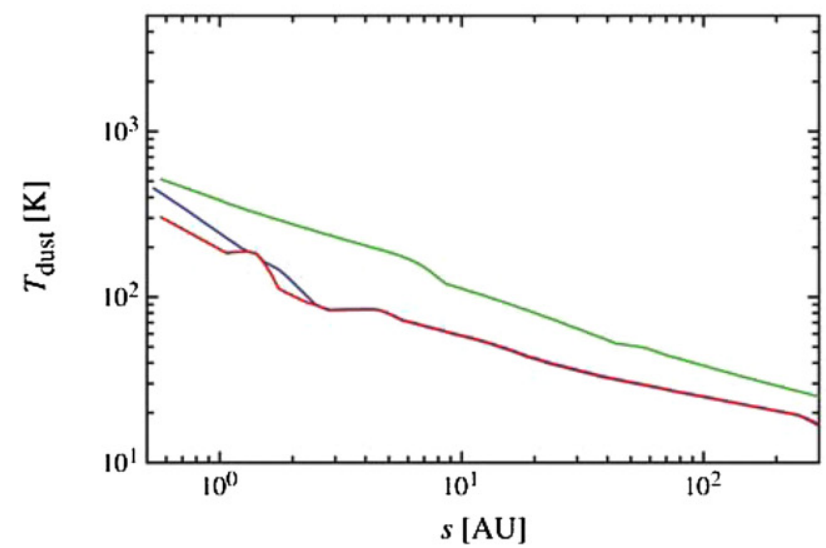

Figure 1. From top to bottom: gas density, gas temperature, and dust temperature for the full disk model (left) and for constant streamlines $z / h$ of the viscous accretion (right), where $h$ denotes the scale height of the disk at radius $s$. For the gas temperature, inlaid are the vertical profiles as a function of $z / s$ in the inner disk region. (A color version of this figure is available in the online journal.)

and $n_{\text {dust }} \pi\left\langle a^{2}\right\rangle$ is the cross section of the dust grains per unit volume, with $n_{\text {dust }} \sim 6 \times 10^{-10} n_{\text {tot }}$ and $\left\langle a^{2}\right\rangle=4.84 \times 10^{-12} \mathrm{~cm}^{2}$ for the dust grain model adopted here. Further, $n_{\text {tot }}$ and $n_{\mathrm{H}}$ denote the total number density of hydrogen nuclei and the number density of atomic hydrogen, respectively. The total number density of hydrogen nuclei is calculated from the mass density, $\rho_{\text {gas }}$, using

$$
n_{\mathrm{tot}}=\frac{\rho_{\mathrm{gas}}}{\left(1+4 y_{\mathrm{He}}\right) m_{\mathrm{H}}}=\frac{\rho_{\mathrm{gas}}}{1.4 m_{\mathrm{H}}},
$$

where $y_{\mathrm{He}}=0.1$ is the fractional elemental abundance of helium. Here, we neglect the mass contained in other, less abundant elements.

Alternatively, in model $\mathrm{B}$, the $\mathrm{H}_{2}$ grain formation is calculated according to Cazaux \& Tielens (2002),

$$
\begin{aligned}
\dot{n}\left(\mathrm{H}_{2}\right)_{\mathrm{B}}^{\mathrm{gr}} & =\dot{n}\left(\mathrm{H}_{2}\right)_{\mathrm{A}}^{\mathrm{gr}} \cdot \varepsilon\left(T_{\text {dust }}\right) \\
& =\frac{1}{2} n_{\mathrm{H}} \cdot v_{\mathrm{H}}^{\mathrm{th}}\left(T_{\text {gas }}\right) n_{\text {dust }} \pi\left\langle a^{2}\right\rangle \alpha_{\mathrm{H}} \varepsilon\left(T_{\text {dust }}\right),
\end{aligned}
$$


Table 1

Molecular Binding Energies $E^{\text {bind }}(\mathrm{K})$

\begin{tabular}{lcc}
\hline \hline Species & $E^{\text {bind }}(\mathrm{K})$ & Reference \\
\hline $\mathrm{H}$ & 350 & $(5)$ \\
$\mathrm{H}_{2}$ & 450 & $(5)$ \\
$\mathrm{CO}_{2}$ & 2690 & $(3)$ \\
$\mathrm{H}_{2} \mathrm{O}$ & 4820 & $(4)$ \\
$\mathrm{CO}$ & 960 & $(1)$ \\
$\mathrm{OH}$ & 1260 & $(5)$ \\
$\mathrm{CH}_{4}$ & 1080 & $(2)$ \\
$\mathrm{NH}_{3}$ & 3080 & $(4)$ \\
$\mathrm{HCN}$ & 4170 & $(2)$ \\
$\mathrm{CH}_{3} \mathrm{OH}$ & 2140 & $(5)$ \\
$\mathrm{C}_{2} \mathrm{H}_{2}$ & 2400 & $(2)$ \\
$\mathrm{SO}_{2}$ & 3070 & $(5)$ \\
$\mathrm{H}_{2} \mathrm{~S}$ & 1800 & $(5)$ \\
\hline
\end{tabular}

References. (1) Sandford \& Allamondola 1988; (2) Yamamoto et al. 1983; (3) Sandford \& Allamondola 1990; (4) Sandford \& Allamondola 1993; (5) Hasegawa \& Herbst 1993.

with several updates for the efficiency $\varepsilon\left(T_{\text {dust }}\right)$ (P. Woitke 2009, private communication). The sticking coefficient, $\alpha_{\mathrm{H}}$, is taken from Hollenbach \& McKee (1979) and approximated to first order, i.e.,

$$
\alpha_{\mathrm{H}} \approx \frac{1}{1+0.4 \sqrt{\left.\left(T_{\mathrm{gas}}+T_{\mathrm{dust}}\right) / 100 \mathrm{~K}\right)}} .
$$

The model for adsorption of gaseous species onto dust grains and desorption of ice species from dust grains is taken from Woitke et al. (2009a), who consider collisional adsorption together with thermal desorption, cosmic-ray-induced desorption, and UV photodesorption.

We calculate the ice formation rates for all neutral and negatively charged species $k$ in our reaction network,

$$
\dot{n}_{k \text { ice }}=n_{k} R_{k}^{\mathrm{ads}}-n_{k \text { ice }}^{\text {desorb }} \cdot\left(R_{k}^{\mathrm{des}, \text { th }}+R_{k}^{\mathrm{des}, \mathrm{cr}}+R_{k}^{\mathrm{des}, \mathrm{ph}}\right),
$$

where $n_{k \text { ice }}$ denotes the number density of ice species $k$ and $n_{k \text { ice }}^{\text {desorb }}$ its fraction located in the uppermost surface layers of the ice mantle. This fraction $n_{k \text { ice }}^{\text {desorb }}$ is given by (Aikawa et al. 1996)

$$
n_{k \text { ice }}^{\text {desorb }}= \begin{cases}n_{k \text { ice }} & n_{\text {ice }}<n_{\text {act }} \\ n_{\text {act }} \frac{n_{k \text { ice }}}{n_{\text {ice }}} & n_{\text {ice }} \geqslant n_{\text {act }},\end{cases}
$$

with $n_{\text {act }}=3 \times 10^{15} \cdot 4 \pi\left\langle a^{2}\right\rangle n_{\text {dust }}$ being the number density of active spots in the ice mantle, and $n_{\text {ice }}$ being the number density of all ice species $\left(n_{\text {tot }}=n_{\text {gas }}+n_{\text {ice }}\right)$. In the following, we summarize the expressions for the rate coefficients and we refer readers to Woitke et al. (2009a) for a detailed discussion.

The adsorption rate for species $k$ with mass $m_{k}$ and thermal velocity $v_{k}^{\text {th }}$ is

$$
R_{k}^{\mathrm{ads}}=\pi\left\langle a^{2}\right\rangle n_{\mathrm{dust}} \alpha_{k} v_{k}^{\text {th }},
$$

where we assume a sticking coefficient of unity for all species excepting hydrogen (cf. $\alpha_{\mathrm{H}}$ in Equation (5)). The desorption rates are

$$
R_{k}^{\text {des, th }}=v_{k}^{\text {osc }} \exp \left(-\frac{E_{k}^{\text {bind }}}{T_{\text {dust }}}\right),
$$

Table 2

Initial Fractional Abundances in the Chemical Network, Including the Gas and Ice Component of Each Species

\begin{tabular}{lc}
\hline \hline Species & $x_{k, 0}{ }^{\mathrm{a}}$ \\
\hline $\mathrm{H}$ & $2.00 \mathrm{e}-07$ \\
$\mathrm{H}^{+}$ & $1.00 \mathrm{e}-10$ \\
$\mathrm{H}_{3}^{+}$ & $1.00 \mathrm{e}-08$ \\
$\mathrm{He}^{+}$ & $2.50 \mathrm{e}-11$ \\
$\mathrm{He}$ & $1.00 \mathrm{e}-01$ \\
$\mathrm{CH}_{4}$ & $2.00 \mathrm{e}-07$ \\
$\mathrm{NH}_{3}$ & $1.00 \mathrm{e}-05$ \\
$\mathrm{H}_{2} \mathrm{O}$ & $1.00 \mathrm{e}-05$ \\
$\mathrm{C}_{2} \mathrm{H}_{2}$ & $5.00 \mathrm{e}-07$ \\
$\mathrm{Si}$ & $3.60 \mathrm{e}-08$ \\
$\mathrm{~N}_{2}$ & $2.00 \mathrm{e}-05$ \\
$\mathrm{C}_{2} \mathrm{H}_{4}$ & $1.00 \mathrm{e}-08$ \\
$\mathrm{CO}$ & $5.00 \mathrm{e}-05$ \\
$\mathrm{H}_{2} \mathrm{CO}$ & $4.00 \mathrm{e}-08$ \\
$\mathrm{CH}_{3} \mathrm{OH}$ & $5.00 \mathrm{e}-07$ \\
$\mathrm{O}_{2}$ & $1.00 \mathrm{e}-06$ \\
$\mathrm{H}_{2} \mathrm{~S}$ & $1.00 \mathrm{e}-06$ \\
$\mathrm{Fe}^{+}$ & $2.40 \mathrm{e}-08$ \\
\end{tabular}

Note. ${ }^{a}$ The initial fractional abundance is defined as $x_{k, 0}=n_{k, 0} / n_{\text {tot }}$.

$$
\begin{gathered}
R_{k}^{\mathrm{des}, \mathrm{cr}}=f_{70 \mathrm{~K}} R_{k, 70 \mathrm{~K}}^{\mathrm{des}, \text { th }} \frac{\zeta_{\mathrm{CR}}}{5 \times 10^{-17} \mathrm{~s}^{-1}}, \\
R_{k}^{\mathrm{des}, \mathrm{ph}}=\pi\left\langle a^{2}\right\rangle \frac{n_{\mathrm{dust}}}{n_{\mathrm{act}}} Y_{k} F_{\mathrm{FUV}} .
\end{gathered}
$$

The binding energies $E_{k}^{\text {bind }}(\mathrm{K})$ for the most important species are listed in Table 1 . The vibrational frequency of species $k$ in the surface potential well is

$$
v_{k}^{\mathrm{osc}}=\left(\frac{3 \times 10^{15} k_{\mathrm{B}} E_{k}^{\mathrm{bind}}}{\pi^{2} m_{k}}\right)^{1 / 2} .
$$

The expression for cosmic-ray-induced desorption is adopted from Hasegawa \& Herbst (1993), who evaluate the rate coefficient at a temperature of $70 \mathrm{~K}$ with a duty cycle of the grains of $f_{70}=3.16 \times 10^{-19}$. The photodesorption yields $Y_{k}$ are simply set to $10^{-3}$ per UV photon (Westley et al. 1995). Finally, the cosmic-ray ionization rate $\zeta_{\mathrm{CR}}$ and the FUV field $F_{\mathrm{FUV}}$ in units of photons $\mathrm{cm}^{-2} \mathrm{~s}^{-1}$ are calculated from the density profile and the dust opacity adopted in the disk model (Nomura et al. 2007). The calculation of the FUV field is based on Nomura \& Millar (2005, Equations (6) and (7)) and accounts for dust scattering.

\subsection{Initial Conditions}

The initial abundances we use as input are listed in Table 2 and we calculate the chemical evolution over $10^{6} \mathrm{yr}$, the typical lifetime of protoplanetary disks. The initial abundances for each species $n_{k, k \text { ice, } 0}$ include both the ice and the gas component.

We compare the freeze-out timescale $\tau_{k}^{\text {ads }}$ and evaporation timescale $\tau_{k}^{\text {des }}$ for each species in a simple model to determine whether it exists initially as gas or ice only:

$$
\tau_{k}^{\mathrm{ads}}=\left(R_{k}^{\mathrm{ads}}\right)^{-1}, \quad \tau_{k}^{\mathrm{des}}=\left(R_{k}^{\mathrm{des}}\right)^{-1} .
$$

For $\tau_{k}^{\text {ads }}<\tau_{k}^{\text {des }}$, the species is initially frozen out, $n_{k \text { ice }}=n_{k, 0}$ and $n_{k}=0$. Otherwise, it exists in the gas phase only, $n_{k}=n_{k, 0}$ and $n_{k \text { ice }}=0$. 


\subsection{Physicochemical Interactions: Viscous Accretion, Turbulent Mixing, and Disk Winds}

Modeling the physicochemical interaction is the key aspect of our study. Even with the steady increase in computational power, this is still a challenging problem which can only be overcome by massive parallel computing and by accepting several simplifications. In the context of a steady disk model, for example, it is a reasonable assumption to describe the interaction between the disk physics and chemistry as a one-way process. Hence, we consider the influence of physical processes on the disk chemistry, but neglect the feedback of the chemistry on the physical disk structure.

Despite this simplification, the calculation of the disk's chemical evolution with interaction is still considerably more computationally expensive, the reason being that adjacent grid cells are chemically coupled through the radial and vertical exchange of material. Accordingly, their evolution must be calculated in parallel with integration time steps that can be shorter than the timescale of the chemical reaction network, due to the Courant-Friedrichs-Lewy (CFL) condition (Courant et al. 1928) of the fluid (see Section 2.6.4). More precisely, to compute the models with interaction, we adopt the following procedure: all grid cells are initialized at the starting time with their initial conditions (Section 2.5) and the transfer coefficients between the grid cells are evaluated (see below for the calculation of these coefficients for the different transport processes). The chemical evolution of the entire disk is calculated in parallel for time steps that satisfy the CFL condition and the transport coefficients are updated each iteration. This step is repeated until a total age of the disk of $10^{6} \mathrm{yr}$ is reached.

In the following, we describe each process we consider in this study. A detailed discussion of the numerical methods used to compute the chemical evolution is given in Appendix A.

\subsubsection{Radial Transport by Viscous Accretion}

First, we allow for the radial inward transport of material through the disk via accretion. The main parameter describing this effect is the accretion rate $\dot{M}=10^{-8} M_{\odot} \mathrm{yr}^{-1}$, which relates the radial inflow velocity $v_{s}$ with the column density $\Sigma$ at radius $s$,

$$
\dot{M}=2 \pi s v_{s} \Sigma \quad\left(v_{s}>0 \text { for radial inflow }\right) .
$$

We assume that inward accretion occurs along streamlines $l=\sqrt{s^{2}+z^{2}}$, with $z / h=$ const. Here, $h$ is the scale height of the disk defined by $h=c_{\mathrm{S}} / \Omega_{\mathrm{K}}, c_{\mathrm{S}}$ is the sound speed at the disk midplane, and $\Omega_{\mathrm{K}}$ is the Keplerian angular frequency.

We denote the net accretion rate of species $k$, i.e., the net change in its number density, with $\dot{n}_{k \text {, acc }}$. In steady state, the net accretion rate of species $k$ can be written as (see Appendix A.1 for the numerical implementation)

$$
\dot{n}_{k, \text { acc }}=\frac{\partial\left(\tilde{A} n_{k} v_{l}\right)}{\partial l},
$$

where $\tilde{A}$ is a geometrical factor and corresponds to $\rho_{i+1} / \rho_{i}$ in (A2). The boundary conditions for the accretion process at the inner and outer disk radii are set as follows: due to the purely inward motion, all material that flows inward from the innermost grid cell is removed from the disk, whereas at the outer radius $s=300 \mathrm{AU}$, the accretion timescales become sufficiently long that we can safely assume $\dot{n}_{k \text {, acc }}=0$ for all species. In Section 2.6.4, we compare the timescales for viscous accretion with the chemical timescale and also with corresponding values for the other physical processes considered here.

\subsubsection{Vertical Transport by Turbulent Mixing}

Although the mechanism is not yet fully clear, it is commonly understood that the source of viscosity in astrophysical disks is turbulence. Hence, a sufficiently large viscosity as is the case in our model $(\alpha=0.01)$ implies that the effect of turbulent mixing is strong, particularly in the inner disk region. While this turbulent mixing, in principle, occurs in the radial and vertical directions, we ignore the radial turbulent motion and focus on the vertical mass fluxes only. Furthermore, we assume that the turbulent velocity is independent of the distance $z$ from the midplane and thus only a function of radius.

We calculate the mixing in a diffusion-type approach. The diffusion coefficient is set as $K=v_{z} d z$, where

$$
v_{z}=v_{z, \operatorname{mix}}=\alpha^{\prime} c_{\mathrm{s}}
$$

in analogy to the expression for the turbulent velocity in a Shakura-Sunyaev disk model, where the turbulent viscosity is

$$
v_{\text {turb }}=v_{\text {turb }} l_{\text {turb }}=\alpha c_{\mathrm{s}} h .
$$

We assume $\alpha^{\prime}=10^{-3}$ to account for the turbulence in vertical direction only. For our numerical model, the length scale $d z$ corresponds to the vertical extent of the grid cells $\Delta z \approx h / 30$. The mixing timescales are discussed in Section 2.6.4.

The usual diffusion equation for species $k$ (cf. Equation (3) in Willacy et al. 2006) reduces to

$$
\dot{n}_{k, \text { mix }}=\frac{\partial}{\partial z}\left(K n_{\mathrm{tot}} \frac{\partial\left(n_{k} / n_{\mathrm{tot}}\right)}{\partial z}\right) .
$$

In Appendix A.2, we describe the numerical implementation of calculating the chemistry accounting for the mixing rates.

The boundary conditions are set at the disk midplane and surface. Due to the symmetry with respect to the midplane, the net exchange rate at $z=0$ is zero. Likewise, we assume that there is no exchange of material between the uppermost disk layer and any potential atmosphere on top of it.

\subsubsection{Vertical Transport by Disk Winds}

We consider vertical disk winds as an alternative description of the vertical motion in the chemical model of the disk. Contrary to turbulent mixing, which implies upward and downward motion, the disk wind transports material upward only (cf. Figure 11(c) in Appendix A.2).

We refer to the wind solutions of Suzuki \& Inutsuka (2009) to describe the vertical disk wind in our protoplanetary disk model. They investigated disk winds driven by MHD turbulence in local three-dimensional MHD simulations of stratified accretion disks and found that large-scale channel flows develop most effectively at 1.5-2 disk scale heights, where the magnetic pressure is comparable to the gas pressure (here we refer to their model with initial plasma $\beta=10^{6}$ ). The breakup of these channel flows drives structured disk winds, which might play an essential role in the dynamical dispersal of protoplanetary disks. While Suzuki \& Inutsuka (2009) perform local threedimensional simulations at radius $s=1$ AU which lead to quasiperiodic cycles of 5-10 rotations and strong mass outflows, our disk model is steady and does not allow for a dispersal of the disk.

We therefore adopt their solutions for the wind velocity $\hat{v}_{\text {wind, } 0}$ at the midplane at $1 \mathrm{AU}$ and calculate the radial and vertical 

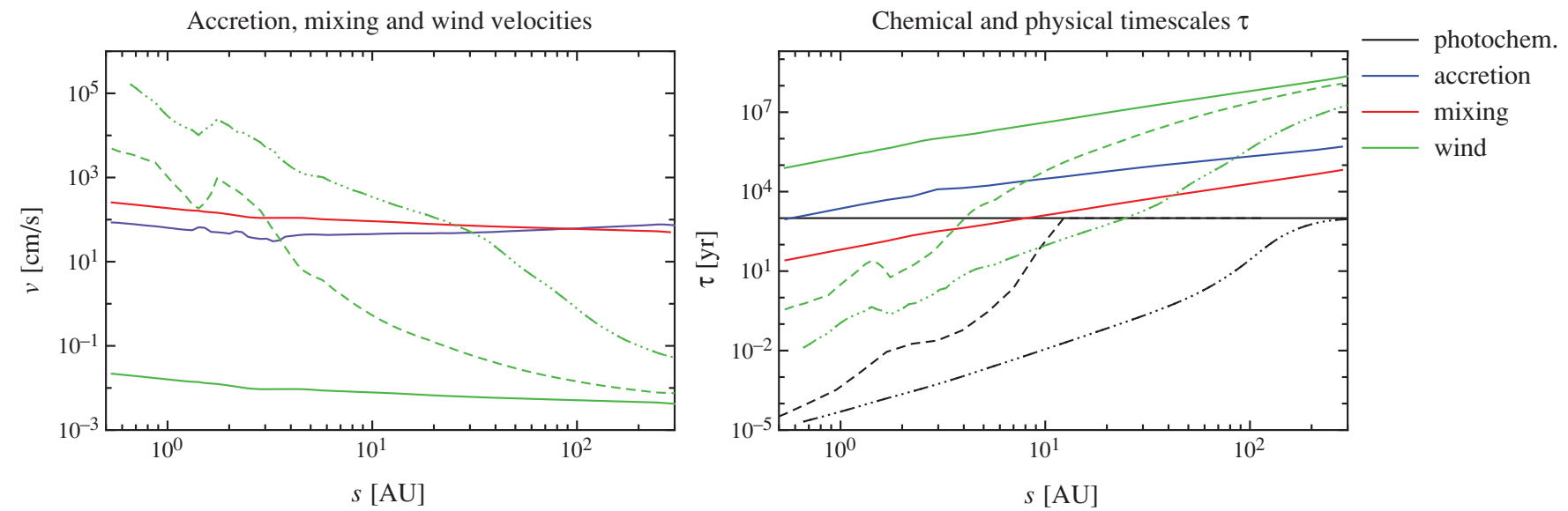

Figure 2. Velocities (left) and timescales (right) for the transport processes of accretion (radial), mixing (vertical), and wind (vertical). Typical timescales of the photochemical reactions are also shown in the right panel (see the text for details). Solid lines denote midplane values, i.e., $z / s=0$, (accretion, mixing, wind), or the timescale of the photochemistry for the interstellar radiation field (photochem.), respectively. Dashed lines correspond to $z / s=0.2$ and dot-dashed lines to $z / s=0.5$.

(A color version of this figure is available in the online journal.)

profile according to Suzuki \& Inutsuka (2009) and the vertical continuity equation in steady state, $\partial\left(\rho v_{z}\right) / \partial z=0$,

$$
\begin{aligned}
& v_{0}=v_{\text {wind }}(s, z=0)=\varkappa \hat{v}_{\text {wind }, 0} \cdot \frac{c_{\mathrm{s}}(s)}{c_{\mathrm{s}}(1 \mathrm{AU})}, \\
& v_{z}=v_{\text {wind }}(s, z)=v_{0} \cdot \frac{\rho(s, z=0)}{\rho(s, z)},
\end{aligned}
$$

where $\hat{v}_{\text {wind }, 0}=8.5 \times 10^{-5} c_{\mathrm{s}}(1 \mathrm{AU})$ (Suzuki \& Inutsuka 2009). For large values of $\hat{v}_{\text {wind }, 0}$, the midplane layers disperse over timescales shorter than the typical disk lifetime of $10^{6} \mathrm{yr}$, which contradicts the assumption of a steady disk model. We therefore introduce an ad hoc factor $\varkappa=10^{-3}$ in Equation (19) to reduce the wind velocities such that the dispersal timescales, $\Sigma /\left(\rho_{\mathrm{gas}} v_{z}\right)$ (where $\Sigma$ is the surface density of the disk), become $\sim 10^{6} \mathrm{yr}$. The resulting wind profiles reproduce the results of Suzuki \& Inutsuka (2009) in the sense that the disk wind is most effective at $\sim 2$ disk scale heights, where the gas density and therefore the gas pressure decrease steeply and any potential magnetic pressure would become comparable with it.

The wind rates are calculated from the vertical continuity equation for species $k$, using the wind velocity profile (Equation (19)):

$$
\dot{n}_{k, \text { wind }}=-\frac{\partial\left(n_{k} v_{z}\right)}{\partial z} .
$$

The wind rates for the discrete grid of the disk are given in Appendix A.3.

The boundary conditions are set as follows: since we adopt small wind velocities so that the disk dispersal time becomes long enough, we can assume that the mass loss is negligible at the midplane and therefore $\dot{n}_{k \text {, wind }}=0$, whereas at the upper boundary, we simply allow all material transported by the disk wind to be lost.

\subsubsection{Timescales}

The grid crossing times for the mass transport by viscous accretion, turbulent mixing, and disk winds are displayed in Figure 2. These are set by the CFL condition for the discrete grid of our disk model:

$$
\tau_{\mathrm{acc}}=\frac{\Delta s}{v_{s}}, \quad \tau_{\mathrm{mix}}=\frac{\Delta z}{v_{z, \text { mix }}}, \quad \tau_{\mathrm{wind}}=\frac{\Delta z}{v_{z, \text { wind }}} .
$$

The radial and vertical size of the grid cells are given by $\Delta s$ and $\Delta z$, respectively, and depend on distance $s$ from the star. For reference, we display the accretion, mixing, and wind velocities in Figure 2 (left panel).

In the right panel of Figure 2, we also plot the timescale of photochemistry given by (Duley \& Williams 1984)

$$
\tau_{\text {photochem }}=10^{3} \mathrm{yr} \cdot \frac{F_{\mathrm{FUV}, \mathrm{ISRF}}}{F_{\mathrm{FUV}}(s, z)},
$$

where $F_{\mathrm{FUV}, \text { ISRF }}$ is the FUV photon flux of the interstellar radiation field. In the upper layers of the disk, the UV radiation field is strong and the timescale for the photochemistry is shorter than the dynamical timescales. Below the transition layer, $z / s \lesssim 0.2$, the incident FUV radiation is highly attenuated and the chemical timescales are controlled by two-body reactions, which are sensitive to the temperature and density. The chemical timescale in this region is generally longer than the photochemical timescale for the interstellar radiation field, $10^{3} \mathrm{yr}$. The accretion and mixing timescales become comparable to this limit at 0.5 and $10 \mathrm{AU}$, respectively. The disk wind timescales are longer than the chemical timescales for all $z / s$ for the entire disk in this model.

\subsection{Infrared Molecular Line Spectra}

Using the results of our chemical evolution calculations, we compute emergent mid-infrared spectra from the disk. We include the dust continuum, which is the dominant source of opacity in protoplanetary disks, along with molecular line emission and absorption, assuming LTE.

The dust continuum opacities are taken from the Jena opacity tables, using routines contained within the RATRAN package (Ossenkopf \& Henning 1994; Hogerheijde \& van der Tak 2000). In accordance with the dust properties of the disk model, we use the tables for spherical dust grains (parameter "e5" in the RATRAN routines) with thin ice coating (parameter "thin").

The HITRAN'2004 molecular spectroscopic database (Rothman et al. 2005) provides a large set of energy levels and line transitions in the infrared for 39 molecules, focusing on terrestrial atmospheric species. Nevertheless, the overlap of species of interest between the HITRAN and UMIST databases is sufficient for our purposes. Since isotopes are not currently included in our chemical reaction network, we neglect spectroscopic information for minor isotopes in the HITRAN database. 
The major issue concerning the calculation of line intensities in the infrared is the limited availability of collisional coefficients, in particular, for the large set of levels and transitions contained in the HITRAN database (e.g., more than 180,000 transitions for the main isotope of $\mathrm{CH}_{4}$ for wavelengths between 1 and $40 \mu \mathrm{m}$ ). Since the main purpose of this work is the calculation of the disk's chemical evolution, a detailed non-LTE radiative transfer calculation of the emergent spectra is beyond the scope of this paper. We therefore assume local thermal equilibrium to model the line emission and absorption strengths for the species of interest. For a species $k$, the transition $j \rightarrow i$ $\left(E_{i}<E_{j}\right)$ is calculated from the standard equations

$$
\begin{gathered}
Q_{k}\left(T_{\text {gas }}\right)=\sum_{i} g_{k, i} \exp \left(-\frac{E_{k, i}}{T_{\text {gas }}}\right), \\
n_{k, i / j}=n_{k} \frac{g_{k, i / j}}{Q_{k}\left(T_{\text {gas }}\right)} \cdot \exp \left(-\frac{E_{k, i / j}}{T_{\text {gas }}}\right), \\
j_{k, i j}=h v A_{k, j i} n_{k, j} \mathcal{V}_{k}, \\
\kappa_{k, i j}=h v\left(B_{k, i j} n_{k, i}-B_{k, j i} n_{k, j}\right) \mathcal{V}_{k} .
\end{gathered}
$$

Here, $n, j$, and $\kappa$ denote the level populations, the line emissivity, and the line opacity, respectively. The Einstein coefficients $A$ and $B$ are listed in the HITRAN database or determined using the principle of detailed balance. The database also provides statistical weights $g$ and partition functions $Q$ for a wide range of temperatures. We calculate Voigt profiles $\mathcal{V}$ including natural line broadening, pressure broadening, and thermal broadening. A Doppler profile at a temperature, $T_{\text {gas }}$, is assumed for the thermal broadening. Turbulent broadening is neglected, since it is negligible compared to thermal broadening for subsonic turbulent velocities. The half-widths for natural broadening $\gamma_{\mathrm{n}}$ and pressure broadening $\gamma_{\mathrm{p}}$ are provided by the HITRAN database at a temperature of $296 \mathrm{~K}$. The temperature dependence of pressure broadening is approximated by

$$
\gamma_{\mathrm{p}}=\gamma_{\mathrm{p}}(296 \mathrm{~K}) \cdot\left(\frac{296 \mathrm{~K}}{T_{\mathrm{gas}}}\right)^{n_{\mathrm{p}}},
$$

where $n_{\mathrm{p}}$ is the temperature-dependent exponent in the HITRAN database.

We adopt a simple, one-dimensional line-of-sight integration to calculate the emergent disk spectra for a disk viewed face-on, i.e., with an inclination $i=0$, relative to the observer. The radial resolution for the spectral calculation is identical to the chemical model. Along each ray, the intensity is integrated following the one-dimensional equation of radiative transfer, accounting for the thermal dust continuum emission and the molecular line emission,

$$
\frac{d I_{v^{\prime}}}{d z}=\left(v^{\prime} / v\right)^{2}\left[j_{v}-\kappa_{\nu} I_{v}\right] .
$$

Quantities on the right-hand side of (27) are evaluated in the moving frame of a fluid element of the observed object, while those on the left-hand side are given in the rest frame of the observer (denoted with '). The observed frequency is given by $v^{\prime}=v(1+(v / c) \cos \theta)$, where $v$ is the velocity of the fluid element and $\theta$ is the angle between the directions of the velocity and the observer. The emission and absorption coefficients are given by

$$
\begin{gathered}
j_{v}=\sum_{k} \sum_{E_{i}<E_{j}} j_{k, i j}+\kappa_{v, \text { dust }} B_{v}\left(T_{\text {dust }}\right), \\
\kappa_{v}=\sum_{k} \sum_{E_{i}<E_{j}} \kappa_{k, i j}+\kappa_{v, \text { dust }}
\end{gathered}
$$

where $B_{v}$ is the Planck function and $\kappa_{\nu, \text { dust }}$ is the specific dust absorption.

The total emission from the disk is calculated as

$$
L_{v^{\prime}, \text { disk }}=8 \pi^{2} \int s d s I_{\nu^{\prime}}(s) .
$$

The full spectrum is obtained from

$$
\begin{aligned}
L_{v^{\prime}} & =L_{v^{\prime}, \text { star }}+L_{v^{\prime}, \text { disk }} \\
& =4 \pi^{2} R^{2} \cdot B_{v^{\prime}}\left(T_{\text {eff }}\right)+L_{v^{\prime}, \text { disk }} .
\end{aligned}
$$

Here, the stellar emission is modeled as blackbody emission with temperature $T_{\text {eff }}=4000 \mathrm{~K}$ and stellar radius $R=2 R_{\odot}$ and is added to the dust continuum and molecular line emission from the disk.

For the line-of-sight integration, a volume-weighting method is adopted to interpolate the temperature and molecular densities before computing the emission and absorption coefficients. In doing so, potential effects of the relatively coarse grid on the emerging line emission are reduced.

\section{RESULTS AND DISCUSSION}

In the following, we discuss the disk chemical evolution results and spectral calculations for the different models considered in this study. First, we investigate the influence of the $\mathrm{H}_{2}$ formation rates on grains on the disk chemistry (Section 3.1). In Sections 3.2 and 3.3, we study the specific effects of viscous accretion, turbulent mixing, and disk winds. Finally, in Section 3.4, we discuss how our results compare with observations of protoplanetary disks.

\section{1. $\mathrm{H}_{2}$ Grain Formation Rates}

Recently, Glassgold et al. (2009) studied the effect of the $\mathrm{H}_{2}$ formation rates on dust grains on the water abundances at the disk surface of the inner disk. We revisit this effect and also study the influence on the infrared molecular line spectra.

In model A, we use the simple approximation from Gould \& Salpeter (1963) and Hollenbach \& Salpeter (1971). In model B, the adopted $\mathrm{H}_{2}$ grain formation rate is that according to Cazaux $\&$ Tielens (2002; see Section 2.4). In both models, the effects of accretion, turbulent mixing, and disk winds are ignored. The chemical evolution of the disk is calculated over $10^{6} \mathrm{yr}$ for a radial range of $0.5-300 \mathrm{AU}$. For both models, the chemical abundances everywhere in the disk have reached steady state by approximately $10^{5} \mathrm{yr}$.

In Figure 3, we show the resulting gas-phase abundances for molecular hydrogen, carbon monoxide, water, and the hydroxyl radical for the inner $100 \mathrm{AU}$ of the disk. In model $\mathrm{B}$, the 

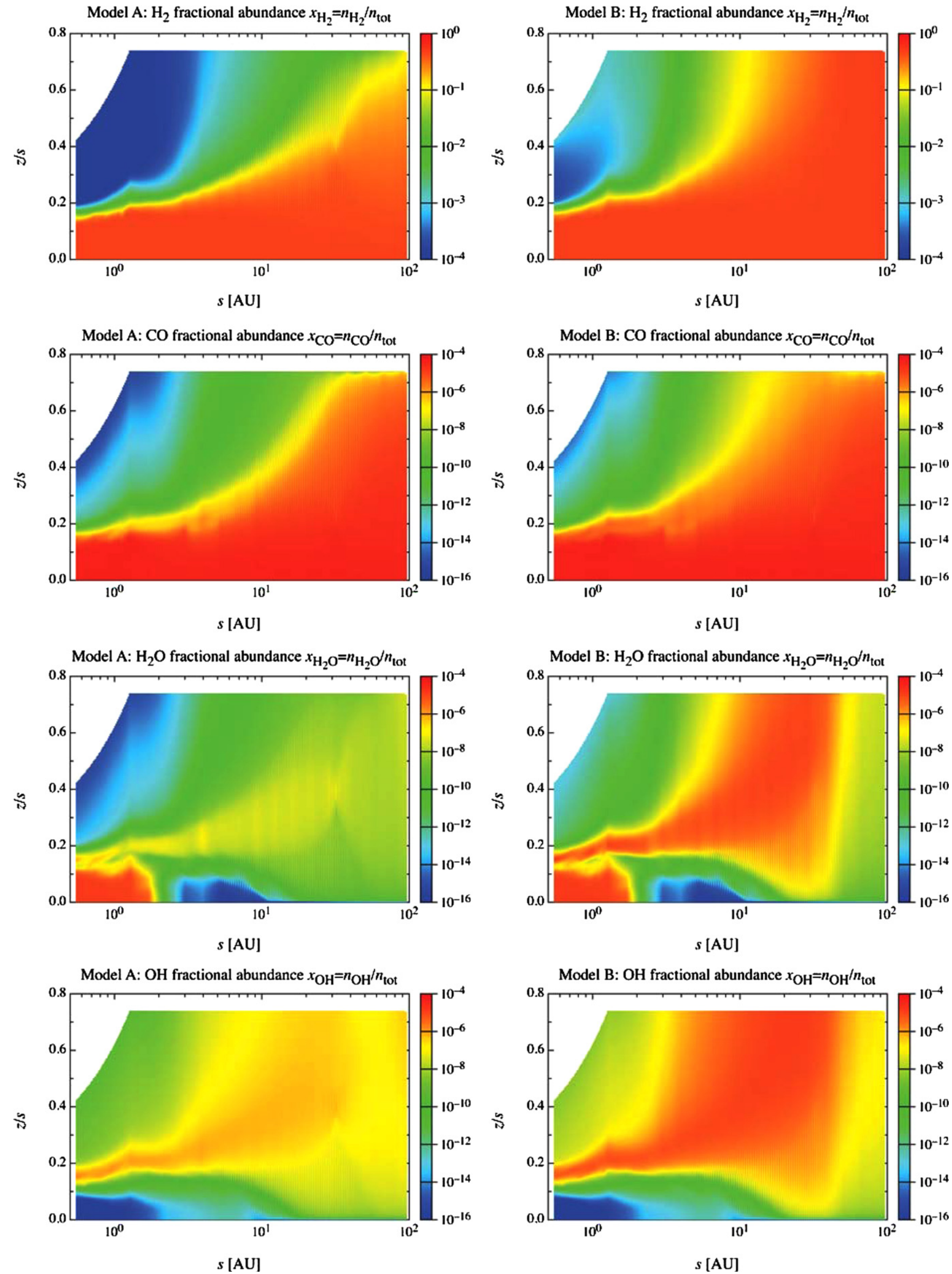

$s[\mathrm{AU}]$

Figure 3. Gas-phase fractional abundances of $\mathrm{H}_{2}, \mathrm{CO}, \mathrm{H}_{2} \mathrm{O}$, and $\mathrm{OH}$ for models $\mathrm{A}$ and $\mathrm{B}$.

(A color version of this figure is available in the online journal.) 

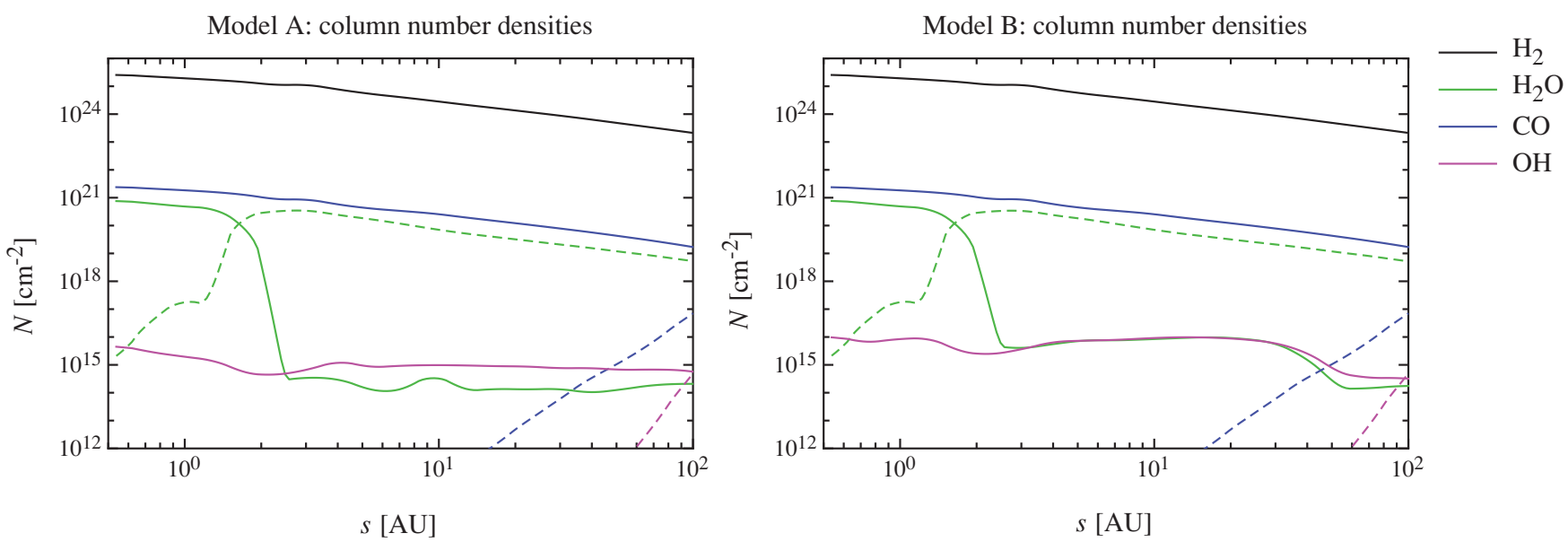

Figure 4. Column densities for the species of interest in models A and B. Solid lines correspond to gas-phase species, dashed lines to ices. (A color version of this figure is available in the online journal.)

formation of molecular hydrogen is more efficient, particularly in the upper layers of the disk. In the warm molecular layer, i.e., the transition layer where the temperature rises and the density decreases steeply, $\mathrm{H}_{2}$ is prone to continuous dissociation due to thermal reactions and incident radiation, and the enhanced grain formation rates in model $\mathrm{B}$ are able to maintain higher fractional abundances. Similarly, in the photodestruction region, i.e., the surface layers of the disk, $\mathrm{H}_{2}$ is more abundant by a factor of $10-50$ between 5 and $100 \mathrm{AU}$. At even smaller radii, the strong stellar UV radiation destroys $\mathrm{H}_{2}$ on timescales shorter than it is formed even in model B. In the lower layers and outer regions of the disk, the differences between models $\mathrm{A}$ and $\mathrm{B}$ become negligible, since here, $\mathrm{H}_{2}$ accounts for essentially all of the hydrogen nuclei.

The differences in the hydrogen chemistry also affect the resulting abundances of predominant molecules in the inner disk, particularly $\mathrm{CO}$ and $\mathrm{H}_{2} \mathrm{O}$. These molecules are commonly found in the planet-forming regions of protoplanetary disks (cf. Salyk et al. 2008). Their modeled abundances depend strongly on the amount of molecular hydrogen at the disk surface and are therefore very different for models $\mathrm{A}$ and $\mathrm{B}$. The increase of molecular hydrogen by a factor of 10-50 between 5 and $100 \mathrm{AU}$ in the upper layers translates to an increase in $\mathrm{CO}$ and $\mathrm{H}_{2} \mathrm{O}$ by a factor of $\approx 1000$. Such a strong correlation is expected theoretically (Glassgold et al. 2009): high fractional abundances of $\mathrm{H}_{2}$ of $\sim 10^{-3}$ in the surface layers produce significant amounts of $\mathrm{CO}, \mathrm{H}_{2} \mathrm{O}$, and $\mathrm{OH}$ through a sequence of neutral radical reactions:

$$
\begin{aligned}
\mathrm{O}+\mathrm{H}_{2} & \longrightarrow \mathrm{H}+\mathrm{OH} \\
\mathrm{OH}+\mathrm{H}_{2} & \longrightarrow \mathrm{H}_{2} \mathrm{O}+\mathrm{H} \\
\mathrm{C}+\mathrm{OH} & \longrightarrow \mathrm{CO}+\mathrm{H} .
\end{aligned}
$$

Glassgold et al. (2009) show that the ratio $x\left(\mathrm{H}_{2} \mathrm{O}\right) / x(\mathrm{O})$ depends quadratically on the ratio $x\left(\mathrm{H}_{2}\right) / x\left(\mathrm{H}^{+}\right)$. Our results confirm this correlation, since the $\mathrm{O}$ and $\mathrm{H}^{+}$abundances are practically identical between models $\mathrm{A}$ and $\mathrm{B}$.

The molecular column densities are only slightly changed compared with the fractional abundances, since the latter are altered only at the disk surface where the density is low (Figure 4). Independent of the $\mathrm{H}_{2}$ formation rate, gaseous $\mathrm{CO}$ traces the total disk mass, with a constant ratio $N_{\mathrm{CO}} / N_{\mathrm{H}_{2}}=10^{-4}$ at radii $<100 \mathrm{AU}$ where the dust temperature is high enough so that $\mathrm{CO}$ does not freeze out. Gas-phase water is fairly abundant at radii $<3 \mathrm{AU}$ (i.e., within the snow line of the disk) with $N_{\mathrm{H}_{2} \mathrm{O}} / N_{\mathrm{CO}}=0.25$. The column density of water is increased by about one order of magnitude between 5 and $50 \mathrm{AU}$, which is due to the large overabundance in the upper layers of model B.

The two $\mathrm{H}_{2}$ formation rate models affect the abundances of the observable molecules in the warm molecular layer and the photodestruction layer, from where infrared molecular line emission originates. In Figure 5, we display the emergent spectra for a disk seen face-on (inclination $i=0$ ). The spectral resolution is set to 600 to match the Spitzer/IRS specifications. The molecular line emission is calculated for the following molecules between 1 and $40 \mu \mathrm{m}$ : $\mathrm{CO}, \mathrm{H}_{2} \mathrm{O}, \mathrm{OH}, \mathrm{CO}_{2}, \mathrm{CH}_{4}$, $\mathrm{NO}, \mathrm{SO}_{2}, \mathrm{NH}_{3}, \mathrm{C}_{2} \mathrm{H}_{2}, \mathrm{~N}_{2}, \mathrm{H}_{2} \mathrm{~S}$, and $\mathrm{NO}^{+}$. The spectra differ substantially between the two models. Model A produces strong and narrow emission lines of $\mathrm{OH}$ between 10 and $40 \mu \mathrm{m}$, due to high abundances in the surface layers of the disk. On the contrary, $\mathrm{H}_{2} \mathrm{O}$ shows broad emission bands between 5 and $10 \mu \mathrm{m}$, but only weak and narrow emission and absorption features at larger wavelengths, in contrast to the observations (cf. Figure 1 in Carr \& Najita 2008). The CO emission band at 4-5 $\mu \mathrm{m}$ is present, as well as an absorption dip of $\mathrm{CO}_{2}$ at $15 \mu \mathrm{m}$. The absorption signatures are generated at radii $\lesssim 2 \mathrm{AU}$, where the temperature increases near the midplane due to viscous heating (cf. the inlay in Figure 1). Since the abundances of $\mathrm{CO}_{2}$ and $\mathrm{H}_{2} \mathrm{O}$ in model $\mathrm{A}$ are high near the disk midplane, but low in the disk surface where the gas temperature increases toward an observer, absorption lines rather than emission lines are generated. Emission or absorption from other molecules included in the spectral calculation (e.g., $\mathrm{NH}_{3}, \mathrm{CH}_{4}$ ) is very weak in the spectrum.

Model $\mathrm{B}$, on the other hand, produces a spectrum with enormous emission features from $\mathrm{OH}, \mathrm{H}_{2} \mathrm{O}$, and $\mathrm{CO}$. The $\mathrm{CO}_{2}$ absorption feature is not affected by the $\mathrm{H}_{2}$ formation model, since it is destroyed very efficiently in the upper layers of the disk, where the main differences in the molecular abundances arise between models $\mathrm{A}$ and $\mathrm{B}$. Comparing the two-dimensional abundances of $\mathrm{OH}, \mathrm{H}_{2} \mathrm{O}$, and $\mathrm{CO}$ in models $\mathrm{A}$ and $\mathrm{B}$ (Figure 3) allows us to constrain the origin of the emission lines to roughly 3-20 AU, where the gas temperature is high enough to contribute to the total flux (cf. Figure 1). Such strong emission features are certainly in disagreement with observations. The enhanced formation rate of $\mathrm{H}_{2}$ on grains from Cazaux \& Tielens (2002) might simply produce too much $\mathrm{H}_{2}$ due to the choice of the variety of parameters used for this model; however, we should 

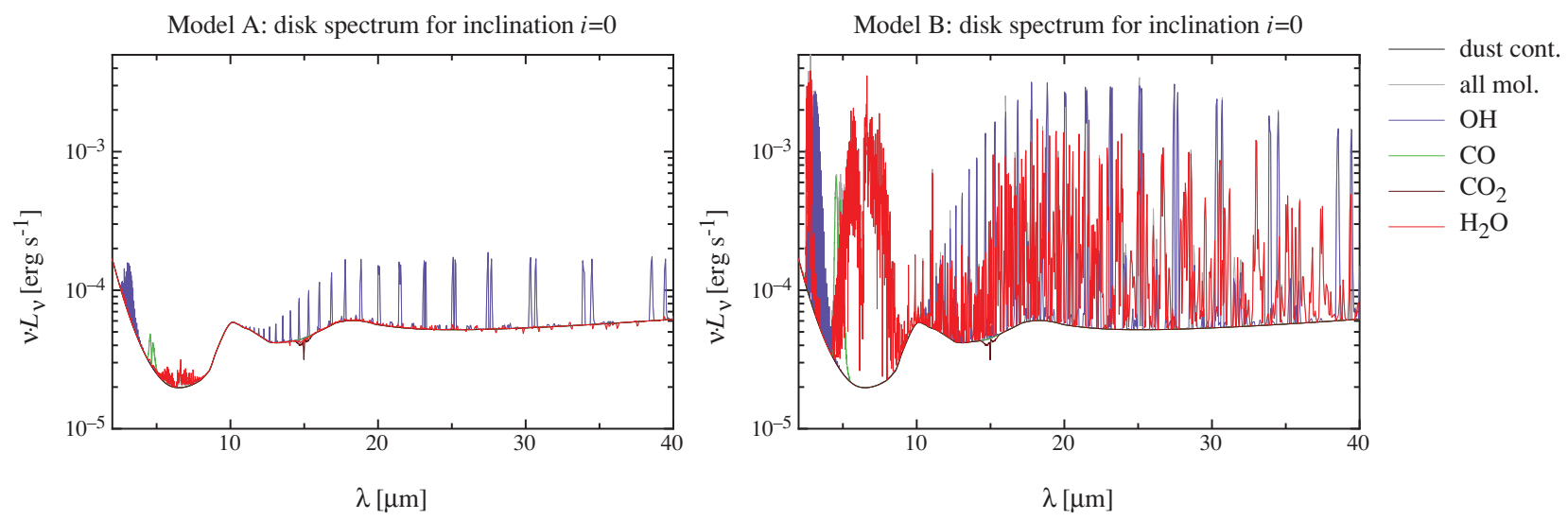

Figure 5. Emergent LTE disk spectra for models A and B for dust continuum and molecular line emission.

(A color version of this figure is available in the online journal.)

note here the limitations of our simple dust model. First, we assume that gas and dust are well mixed in the disk, with a constant mass density ratio of $\rho_{\text {gas }} / \rho_{\text {dust }} \approx 100$. Numerous studies show that dust grains will settle toward the midplane instead of residing in the upper disk layers (see, e.g., Dominik et al. 2007 for a review). Less dust grains in these layers lead to lower $\mathrm{H}_{2}$ abundances and thus to lower abundances of water and OH. Aikawa \& Nomura (2006) demonstrated that less dust grains will also lead to a lower gas temperature at the disk surface, which reduces the line intensities. Since the same line of argument applies to model $\mathrm{A}$, it implies even lower abundances and thus weaker emission lines for $\mathrm{H}_{2} \mathrm{O}$ in this model. Second, the spectral calculations in our study assume LTE level populations, which may overestimate the strength of the emission lines. Meijerink et al. (2009) studied the effects of non-LTE radiative transfer on the molecular emission lines of water: they assume fractional $\mathrm{H}_{2} \mathrm{O}$ abundances between $10^{-10}$ and $10^{-4}$ throughout the disk. Interestingly, they conclude that a strong depletion at $s<1 \mathrm{AU}$ is required to account for observed emission line spectra, and such a depletion is found in our model A. There, the $\mathrm{H}_{2} \mathrm{O}$ abundance is low in the surface layer, because of the efficient destruction of water due to the stellar irradiation and the high temperature. Most important, however, is that Meijerink et al. (2009) find drastic differences between LTE and non-LTE spectral calculations: the LTE calculations overestimate the strength of the emission lines by a factor of 10 for the strongest transitions (i.e., the largest Einstein coefficients). In model B, the line emission is generated in the upper layers of the disk, where the gas number density is of the order of $10^{8} \mathrm{~cm}^{-3}$. The water line transitions between 10 and $40 \mu \mathrm{m}$ reported from Spitzer observations (e.g., Carr \& Najita 2008) are mainly rotational transitions in the ground vibrational state and in the first vibrational state with critical densities of $10^{12} \mathrm{~cm}^{-3}$ or larger (Meijerink et al. 2009). Vibrational transitions between the two states that fall into this wavelength range have even higher critical densities. Hence, the conditions for LTE are partly violated and a proper nonLTE calculation of the disk spectra should be considered. In the following sections, we ignore the enhanced $\mathrm{H}_{2}$ formation rates of model B and study the effects of accretion, turbulent mixing, and wind on model A only.

\subsection{Accretion, Mixing, and Disk Winds-Single Column Solutions}

To investigate the effects of including physicochemical interaction, we first focus on one vertical column of the disk at

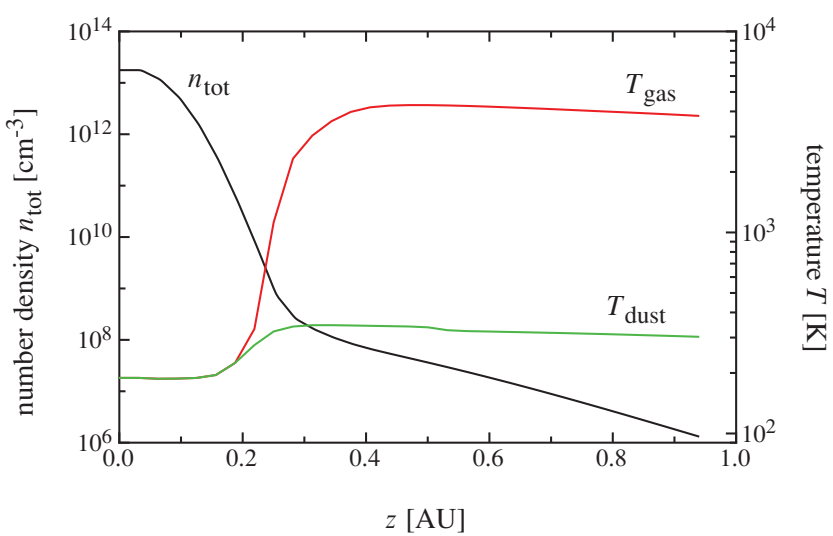

Figure 6. Gas and dust temperatures $T_{\text {gas }}$ and $T_{\text {dust }}$, and total number density $n_{\text {tot }}$ as a function of vertical distance from the disk midplane at a radius $s=1.3 \mathrm{AU}$. (A color version of this figure is available in the online journal.)

radius $s=1.3 \mathrm{AU}$. Here, the temperatures of gas and dust are high enough, in particular in the upper layers of the disk, to allow for an active chemistry. Further, near- and mid-infrared observations derive characteristic radii for molecular line emission around $1 \mathrm{AU}$ (cf. Section 3.4).

In the following, we discuss in detail the modeled molecular abundances for a number of species of interest. For a better understanding, in particular for interpreting the resulting fractional abundances $x_{k}=n_{k} / n_{\text {tot }}$, we display the physical properties of the disk column at this radius in Figure 6. The gas and dust temperatures are tightly coupled in the dense midplane with values around $200 \mathrm{~K}$. Hence, practically all species have desorbed from the dust grains and the ice abundances are negligible. In the warm molecular layer $(z \approx 0.2 \mathrm{AU})$, the gas and dust temperatures begin to decouple due to the lower density. In the photodestruction layer, the gas is heated up to $4000 \mathrm{~K}$ because of the strong stellar irradiation, while the dust temperature is around a factor of 10 lower.

In Figure 7, we plot the fractional abundances as a function of vertical distance from the midplane for the model without physicochemical interaction (model A) and for models with either accretion, turbulent mixing or disk winds (models ACR, MIX, and WND). The gas-phase abundances are shown together with the-mostly negligible-ice abundances. All four models make use of the simple $\mathrm{H}_{2}$ formation rates on grains (Equation (2)), while model B (not displayed) includes the enhanced $\mathrm{H}_{2}$ formation rates on grains (Equation (4)) without physicochemical interaction. Table 3 at the end of this section 

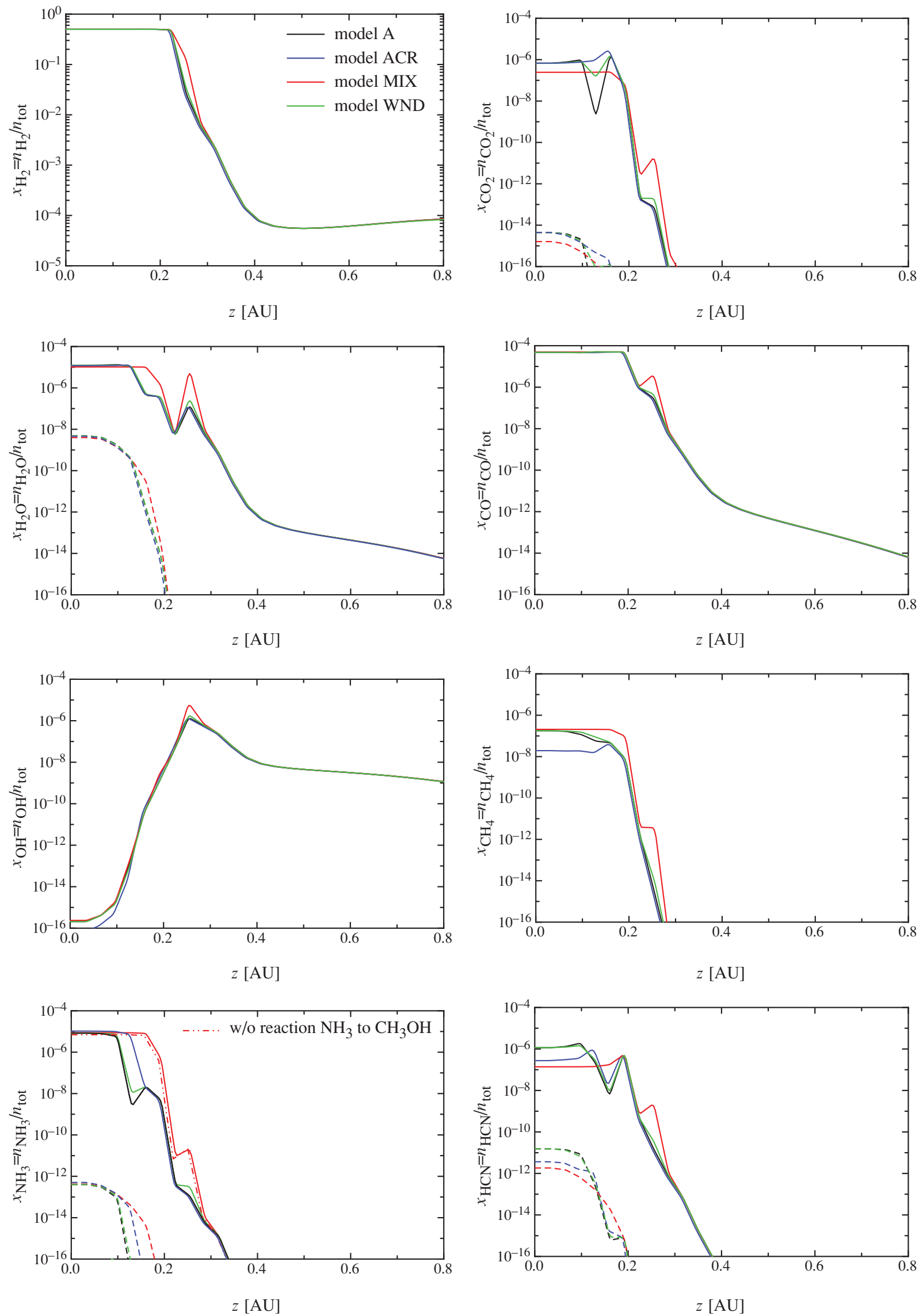

Figure 7. Fractional abundance profiles of gas-phase species (solid) and ices (dashed) as a function of vertical distance from the midplane at $s=1.3 \mathrm{AU}$ in model A (black), and in models with accretion (ACR, blue), mixing (MIX, red), or disk winds (WND, green). Gas and ice fractional abundances at $s=1.3 \mathrm{AU}$.

(A color version of this figure is available in the online journal.) 

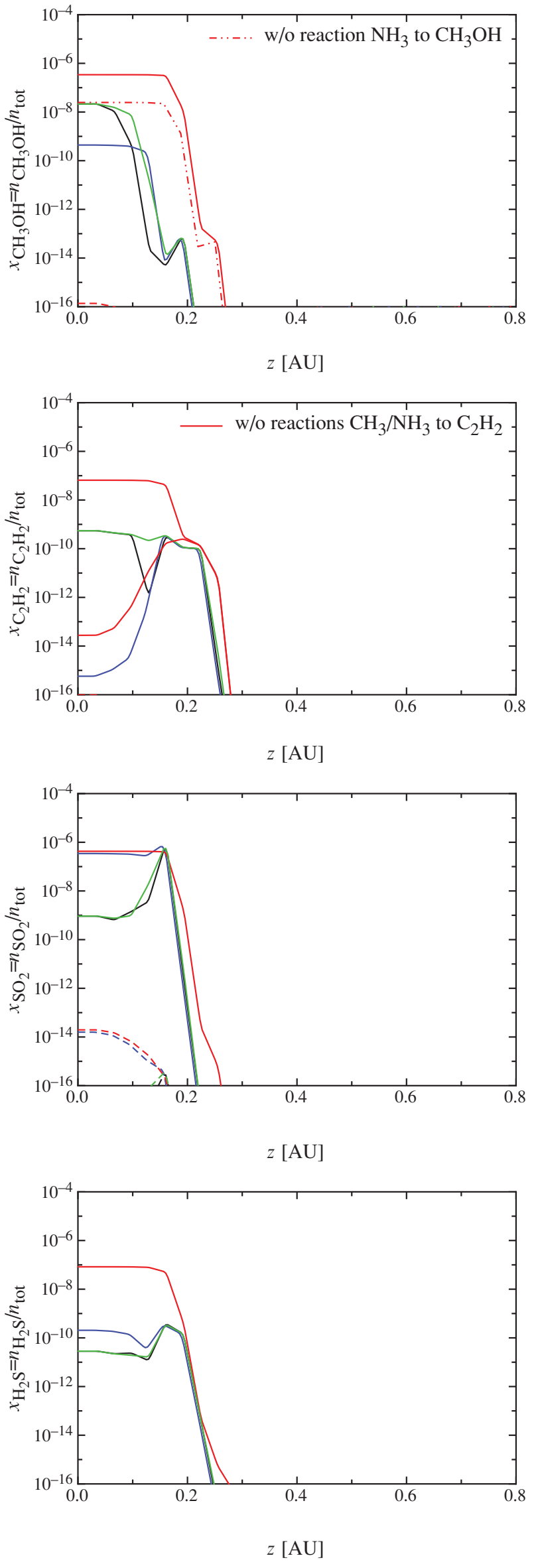

Figure 7. (Continued)
Table 3

Calculated Column Densities $N\left(\mathrm{~cm}^{-2}\right)$ at Radius $s=1.3 \mathrm{AU}$ of Selected Species for the Disk Models Considered in this Study

\begin{tabular}{lccccc}
\hline \hline Species & $\mathrm{A}$ & $\mathrm{B}$ & ACR & MIX & WND \\
\hline $\mathrm{H}_{2}$ & $1.7 \mathrm{e}+25$ & $1.7 \mathrm{e}+25$ & $1.7 \mathrm{e}+25$ & $1.7 \mathrm{e}+25$ & $1.7 \mathrm{e}+25$ \\
$\mathrm{CO}_{2}$ & $2.5 \mathrm{e}+19$ & $2.6 \mathrm{e}+19$ & $2.5 \mathrm{e}+19$ & $8.3 \mathrm{e}+18$ & $2.4 \mathrm{e}+19$ \\
$\mathrm{H}_{2} \mathrm{O}$ & $4.2 \mathrm{e}+20$ & $4.2 \mathrm{e}+20$ & $4.0 \mathrm{e}+20$ & $3.5 \mathrm{e}+20$ & $4.2 \mathrm{e}+20$ \\
$\mathrm{OH}$ & $1.5 \mathrm{e}+15$ & $9.3 \mathrm{e}+15$ & $1.5 \mathrm{e}+15$ & $5.3 \mathrm{e}+15$ & $1.8 \mathrm{e}+15$ \\
$\mathrm{CO}$ & $1.6 \mathrm{e}+21$ & $1.6 \mathrm{e}+21$ & $1.6 \mathrm{e}+21$ & $1.7 \mathrm{e}+21$ & $1.6 \mathrm{e}+21$ \\
$\mathrm{CH}_{4}$ & $5.4 \mathrm{e}+18$ & $5.4 \mathrm{e}+18$ & $6.5 \mathrm{e}+17$ & $7.1 \mathrm{e}+18$ & $5.7 \mathrm{e}+18$ \\
$\mathrm{NH}_{3}$ & $2.5 \mathrm{e}+20$ & $2.5 \mathrm{e}+20$ & $3.4 \mathrm{e}+20$ & $3.0 \mathrm{e}+20$ & $2.6 \mathrm{e}+20$ \\
$\mathrm{HCN}$ & $4.3 \mathrm{e}+19$ & $3.8 \mathrm{e}+19$ & $1.1 \mathrm{e}+19$ & $4.7 \mathrm{e}+18$ & $4.0 \mathrm{e}+19$ \\
$\mathrm{CH}_{3} \mathrm{OH}$ & $4.7 \mathrm{e}+17$ & $4.8 \mathrm{e}+17$ & $1.4 \mathrm{e}+16$ & $1.2 \mathrm{e}+19$ & $5.6 \mathrm{e}+17$ \\
$\mathrm{C}_{2} \mathrm{H}_{2}$ & $1.6 \mathrm{e}+16$ & $1.6 \mathrm{e}+16$ & $1.3 \mathrm{e}+14$ & $2.2 \mathrm{e}+18$ & $1.6 \mathrm{e}+16$ \\
$\mathrm{SO}_{2}$ & $2.7 \mathrm{e}+17$ & $2.6 \mathrm{e}+17$ & $1.2 \mathrm{e}+19$ & $1.4 \mathrm{e}+19$ & $2.9 \mathrm{e}+17$ \\
$\mathrm{H}_{2} \mathrm{~S}$ & $9.6 \mathrm{e}+14$ & $8.3 \mathrm{e}+17$ & $6.2 \mathrm{e}+15$ & $2.8 \mathrm{e}+18$ & $9.4 \mathrm{e}+14$ \\
$\mathrm{OCS}$ & $3.3 \mathrm{e}+19$ & $3.2 \mathrm{e}+19$ & $2.0 \mathrm{e}+19$ & $1.6 \mathrm{e}+19$ & $3.3 \mathrm{e}+19$ \\
\hline
\end{tabular}

compares the column densities of the most important species at radius 1.3 AU for all models A, B, ACR, MIX, and WND. In the following, we discuss in detail the effects of turbulent mixing on the vertical chemical stratification at a radius $s=1.3 \mathrm{AU}$, since this model has the largest effects on the chemical structure of the disk.

\subsubsection{Turbulent Mixing}

Turbulent mixing has important effects on the disk's chemical composition, in particular in the upper layers. Generally, diffusive mixing tries to homogenize the fractional abundances of molecules over the depth of the disk. Figure 7 illustrates this effect; for example, in the case of $\mathrm{H}_{2}$, the fractional abundances are slightly increased in the transition region, with only negligible changes in the column density (cf. Table 3 at the end of this section).

This effect is more pronounced for $\mathrm{CO}_{2}$ : in model $\mathrm{A}, \mathrm{CO}_{2}$ is abundant close to the midplane only, since it is removed from the upper layers by reactions with $\mathrm{H}$ and $\mathrm{H}^{+}$and by photodissociation due to the incident $\mathrm{UV}$ and $\mathrm{X}$-ray radiation

$$
\begin{aligned}
\mathrm{H}+\mathrm{CO}_{2} & \longrightarrow \mathrm{CO}+\mathrm{OH} \\
\mathrm{H}^{+}+\mathrm{CO}_{2} & \longrightarrow \mathrm{HCO}^{+}+\mathrm{O} \\
\mathrm{CO}_{2}+\gamma_{\mathrm{UV}} & \longrightarrow \mathrm{CO}+\mathrm{O} \\
\mathrm{CO}_{2}+\gamma_{\mathrm{X}-\mathrm{ray}} & \longrightarrow \mathrm{CO}+\mathrm{O} .
\end{aligned}
$$

At $z \approx 0.2 \mathrm{AU}$, the reactions with $\mathrm{H}^{+}$and the $\mathrm{X}$-ray photochemistry are dominant, while in the upper layers, the UV photochemistry is most important. In model MIX, $\mathrm{CO}_{2}$ diffuses upward and its abundance is reduced near the midplane.

The $\mathrm{H}_{2} \mathrm{O}, \mathrm{OH}$, and $\mathrm{CO}$ abundances are correlated with that of molecular hydrogen and are enhanced in model MIX in the region where $\mathrm{H}_{2}$ is more abundant. The effect of diffusion of $\mathrm{H}_{2} \mathrm{O}$ leads to reduced fractional abundances for $z<0.15 \mathrm{AU}$ and increased abundances between $0.15 \mathrm{AU}$ and $0.2 \mathrm{AU}$. Water is destroyed easily at high temperatures and through photodissociation by UV and X-ray photons. The $\mathrm{H}_{2} \mathrm{O}$ abundance has a dip at $z \approx 0.22 \mathrm{AU}$, where both the gas temperature and density are relatively low (cf. Woitke et al. 2009b, Figure 1). Here, UV and X-ray photodestruction become strong, while at the same time the temperature is still too low to activate the main formation reactions for $\mathrm{H}_{2} \mathrm{O}$,

$$
\begin{aligned}
\mathrm{H}_{2}+\mathrm{O} & \longrightarrow \mathrm{OH}+\mathrm{H} \\
\mathrm{OH}+\mathrm{H}_{2} & \longrightarrow \mathrm{H}_{2} \mathrm{O}+\mathrm{H} .
\end{aligned}
$$


In the case of methane, diffusion also increases the fractional abundances in the surface layers. At the same time, the formation of $\mathrm{CH}_{4}$ close to the midplane is efficient enough to maintain its initial fractional abundance, leading to an overall increase in the column density of methane. For $z \geqslant 0.3 \mathrm{AU}$, methane is destroyed quickly by UV and X-ray photons.

A similar mechanism is responsible for the considerable increase in ammonia for $z \leqslant 0.3 \mathrm{AU}$. The formation of ammonia near the disk midplane is driven by a change in the oxygen chemistry, with $\mathrm{O}_{2}$ being overabundant in model MIX (see also Section 3.3). The differences in the oxygen abundance between model A and model MIX are caused by the continuous diffusion of $\mathrm{O}_{2}$ from the warm molecular layer toward the midplane. This implies a decrease in the column density of HCN, whose formation is competing with $\mathrm{NH}_{3}$ for the available nitrogen. Since the number of nitrogen atoms over the full column is conserved and since the formation of $\mathrm{NH}_{3}$ is more efficient, $\mathrm{HCN}$ is depleted by one order of magnitude close to the midplane, compared with that in model A. Its column density decreases by approximately the amount required for the formation of the additional ammonia molecules.

The most pronounced effect arises in the case of methanol as a consequence of the increase in ammonia. Its fractional abundance increases for $z \leqslant 0.3 \mathrm{AU}$ by one to six orders of magnitude, resulting in a column density of $1.2 \times 10^{19} \mathrm{~cm}^{-2}$ compared with $4.7 \times 10^{17} \mathrm{~cm}^{-2}$ in model $\mathrm{A}$. This is due to an enhanced production of $\mathrm{CH}_{3} \mathrm{OH}$ by the reaction (Rodgers \& Charnley 2001)

$$
\mathrm{CH}_{3} \mathrm{OH}_{2}^{+}+\mathrm{NH}_{3} \longrightarrow \mathrm{NH}_{4}^{+}+\mathrm{CH}_{3} \mathrm{OH} \text {. }
$$

In Figure 7, we display the ammonia and methanol abundances for an additional model calculation with diffusive mixing, but where this reaction is switched off. The ammonia abundances remain unchanged, but the methanol abundances drop by one order of magnitude, which is comparable to the midplane abundances in model A.

Significant changes are also found for acetylene, $\mathrm{C}_{2} \mathrm{H}_{2}$. At $z=0.2 \mathrm{AU}$, it is mainly produced from $\mathrm{NH}_{3}$ by the reactions

$$
\begin{aligned}
& \mathrm{NH}_{3}+\mathrm{C}_{2} \mathrm{H}_{3}^{+} \longrightarrow \mathrm{C}_{2} \mathrm{H}_{2}+\mathrm{NH}_{4}^{+} \\
& \mathrm{NH}_{3}+\mathrm{C}_{2} \mathrm{H}_{2}^{+} \longrightarrow \mathrm{C}_{2} \mathrm{H}_{2}+\mathrm{NH}_{3}^{+},
\end{aligned}
$$

as well as by a reaction of $\mathrm{C}$ and $\mathrm{CH}_{3}$ at $z<0.2 \mathrm{AU}$,

$$
\mathrm{C}+\mathrm{CH}_{3} \longrightarrow \mathrm{C}_{2} \mathrm{H}_{2}+\mathrm{H} \text {. }
$$

Switching off these reactions leads to a significant decrease in $\mathrm{C}_{2} \mathrm{H}_{2}$ (cf. Figure 7). Hence, acetylene profits from the increase in ammonia abundances and is overabundant by two orders of magnitude at $z \leqslant 0.2 \mathrm{AU}$. Higher up in the disk, it is destroyed efficiently by photochemical reactions and reactions with atomic hydrogen.

Finally, we inspect the sulfur-bearing species $\mathrm{SO}_{2}$ and $\mathrm{H}_{2} \mathrm{~S}$. Initially, sulfur is locked in $\mathrm{H}_{2} \mathrm{~S}$ only, with a fractional abundance of $10^{-6}$ (cf. Table 2). $\mathrm{H}_{2} \mathrm{~S}$ is easily destroyed by atomic hydrogen due to the small activation barrier of this reaction. Provided the $\mathrm{O}_{2}$ and $\mathrm{OH}$ abundances are sufficiently high, this drives a chain of reactions (Charnley 1997; Millar et al. 1997) which forces large amounts of $\mathrm{H}_{2} \mathrm{~S}$ into $\mathrm{SO}_{2}$ in both models at $z \leqslant 0.25 \mathrm{AU}$. The now highly abundant $\mathrm{SO}_{2}$ near the midplane is destroyed on timescales longer than its formation timescales and therefore provides a steady reservoir to maintain the enhanced $\mathrm{H}_{2} \mathrm{~S}$ abundances in comparison to model A. At the midplane, the destruction of $\mathrm{H}_{2} \mathrm{~S}$ is less efficient, but still sufficient to produce OCS from CO and S (Hatchell et al. 1998). After $10^{6} \mathrm{yr}$, sulfur is locked in $\mathrm{SO}_{2}$ and OCS in equal amounts.

Contrarily, in model A, the formation of $\mathrm{SO}_{2}$ is inefficient in the lower layers due to the missing $\mathrm{OH}$ and the missing $\mathrm{O}_{2}$ at $z \approx 0$. Thus, the slower reaction path of $\mathrm{S}$ with $\mathrm{CO}$ is preferred and OCS is built up over the lifetime of the disk, eventually locking all sulfur.

\subsubsection{Disk Winds}

The resulting chemical abundances for model WND are displayed in Figure 7. For all species, the effects of the disk wind on the vertical stratification of the chemical species are much less pronounced than in the case of turbulent mixing. In the cases of $\mathrm{CO}_{2}, \mathrm{NH}_{3}, \mathrm{CH}_{3} \mathrm{OH}$, and $\mathrm{C}_{2} \mathrm{H}_{2}$, slight increases in the fractional abundances are found around $z=0.2 \mathrm{AU}$, in particular where narrow dips occur in the abundances from model A. Protected by the upper layers against the UV and X-ray irradiation, molecules transported upward from the midplane fill these sinks on timescales comparable to their removal by chemical reactions.

The reason for the inefficiency of the disk wind lies in the steady disk model assumption considered here: to avoid disk dispersal over the lifetime of the disk, which would contradict the steady state assumption, we introduced a scaling factor $\varkappa=10^{-3}$ for the resulting wind speeds of Suzuki \& Inutsuka (2009) at the midplane layer. Hence, the upward velocities near the midplane are low in model WND, compared to the turbulent velocities in model MIX (cf. Figure 2). At radii $s \leqslant 10 \mathrm{AU}$, the wind velocities become very large in the photodestruction region of the disk $(z / s>0.3)$, due to the continuity equation in vertical direction in steady state. But there, the timescales of the photochemistry are extremely short so that the effect of the disk wind is small (cf. Figure 2).

For a scaling factor $\varkappa=10^{-3}$, the midplane grid cell at radius $s=1.3 \mathrm{AU}$ evaporates in about $2 \times 10^{5} \mathrm{yr}$, corresponding to a mass loss of $20 \%$ of the total mass contained in this disk ring. Hence, in a stationary model, $x$ should be lower than $10^{-3}$, which would lead to even less efficient disk winds.

We thus conclude that in a steady disk model, the disk winds have only negligible effects. Due to the necessary downscaling of the wind speeds by a factor of $1000\left(\varkappa=10^{-3}\right)$, the vertical mass transport becomes small and causes only small changes in the disk's chemical composition. Nonetheless, it is worth noting that in a time-dependent physical disk model, where evaporation and dynamical refueling by the accretion flow are allowed, the disk wind may become significant. The simple model considered here suggests that it will affect molecules that also show an enhancement in the upper layers in the diffusive mixing model (e.g., $\mathrm{NH}_{3}, \mathrm{CH}_{3} \mathrm{OH}$ ).

\subsubsection{Viscous Accretion}

The chemical abundances from model ACR at a radius of 1.3 AU are displayed in Figure 7. As in the previous cases, at the disk surface photochemistry dominates and the accretion flow does not affect the chemical composition. Close to the disk midplane, the accretion timescale is comparable to the chemical timescale and the effects of the radial inflow of material become visible. Whether the accretion flow leads to an increase or a decrease in the abundance of a particular species depends on the formation and destruction timescales of that species. In the case of $\mathrm{H}_{2} \mathrm{O}$, for example, the chemical reactions are fast enough to compensate for the effects of the accretion flow and 
the abundances and column density are similar to those from model A.

On the contrary, $\mathrm{NH}_{3}$ shows higher abundances at $z<$ 0.15 AU. As discussed for the case of diffusive mixing above, the total amount of nitrogen atoms is conserved and determined by the initial abundances of $\mathrm{N}_{2}$ and $\mathrm{NH}_{3}$ (cf. Table 2). An increase in the column density of $\mathrm{NH}_{3}$ from $2.5 \times 10^{20} \mathrm{~cm}^{-2}$ in model A to $3.4 \times 10^{20} \mathrm{~cm}^{-2}$ in model ACR leads to a decrease in $\mathrm{HCN}$ from $4.3 \times 10^{19} \mathrm{~cm}^{-2}$ to $1.1 \times 10^{19} \mathrm{~cm}^{-2}$ at this radius.

In contrast to the results from model MIX, methanol shows lower abundances close to the disk midplane, despite the increase in ammonia. Since its fractional abundance is even lower than those from model A, the reason, therefore, is not only the lack of vertical diffusion, but the fact that the formation and evaporation timescales (from dust grains) of methanol at around 10 AU are longer than its destruction timescale (cf. Section 3.3). The accretion flow propagates this depletion inward over time. Compared to model A, this reduces the $\mathrm{CH}_{3} \mathrm{OH}$ column density from $4.7 \times 10^{17} \mathrm{~cm}^{-2}$ to $1.4 \times 10^{16} \mathrm{~cm}^{-2}$.

One should bear in mind that the location of the snow line for methanol and hence its gas-phase abundance is sensitive to its binding energy on dust grains, which varies across the literature. Here, we adopt the theoretical value from Hasegawa $\&$ Herbst (1993), $E_{\text {bind }}=2140$ K. Sandford \& Allamondola (1993) and more recently Brown \& Bolina (2007) find much higher values between 4000 and $5000 \mathrm{~K}$ based on the results of temperature-programmed desorption (TPD) experiments on pure methanol ice. Higher binding energies would imply lower gas-phase abundances and a shift of the snow line to smaller radii. Here, the $\mathrm{NH}_{3}$ abundances are higher and the formation of methanol more efficient, which would imply higher gas-phase abundances within the snow line.

A similar decrease in the abundances in the innermost disk can be found particularly for $\mathrm{C}_{2} \mathrm{H}_{2}$, where the accretion flow removes a considerable fraction of acetylene close to the midplane (cf. Table 3 for the resulting column densities of $\mathrm{C}_{2} \mathrm{H}_{2}$ and other species).

\subsection{Accretion, Mixing-Full Disk Solutions}

In the previous section, we inspected in detail the chemical abundances for the different disk models as a function of height at a single radial position. The chemical composition varies between the individual models due to mass transport in the radial and vertical directions. The results thereby depend strongly on the species considered, in particular, on its formation and destruction timescales relative to the dynamical timescales. Since these timescales vary with radial distance from the star for accretion, turbulent mixing, and disk winds, we expect significant changes in the effects of these mass transport phenomena at different radial positions in the disk.

Here, we focus on the radial accretion and vertical mixing and neglect the disk wind. At a radius, $s=1.3 \mathrm{AU}$, the influence of the disk wind on the chemistry is small, compared with diffusive mixing, due to the low wind speed required to impede the evaporation of the disk in our model. At larger radii, the wind velocities are even smaller, leading to longer timescales of the upward wind motion. At the same time, however, the chemical timescales increase (cf. Section 2.6.4), so that we do not find significant changes to the disk chemistry due to the inclusion of a disk wind.

We compare the resulting fractional gas-phase abundances and the gas and ice column densities for model ACR and model MIX with model A in Figure 8. The results for water differ only slightly between the models, and the only difference arises in a narrow strip $z / s \approx 0.2-0.4$, where the gas-phase abundances of water are increased in model MIX due to the enhanced $\mathrm{H}_{2}$ abundances (see also the discussion in Section 3.1). This leads to an increase in the gas-phase column density between 2 and $10 \mathrm{AU}$. Since the $\mathrm{H}_{2} \mathrm{O}$ abundances are increased in the transition layer, turbulent mixing affects the strength of the $\mathrm{H}_{2} \mathrm{O}$ line emission from the disk. This can be seen in Figure 9, where we compare the emerging disk spectra for model A, model ACR, and model MIX. Apart from $\mathrm{H}_{2} \mathrm{O}$, the species dominating the molecular line emission are $\mathrm{CO}$ and $\mathrm{OH}$. Both show slightly stronger emission lines in model MIX and in model ACR, although the effect is more pronounced in the case of diffusive mixing.

In the case of $\mathrm{CO}_{2}$, turbulent mixing reduces the gas-phase column density inside $10 \mathrm{AU}$ : turbulent mixing leads to the diffusion of $\mathrm{CO}_{2}$ from the lower, protected layers into the upper disk, where it is efficiently destroyed by incident radiation, compared with its production. For radii $\leqslant 1 \mathrm{AU}, \mathrm{CO}_{2}$ is strongly depleted. Viscous accretion, on the other hand, enhances the $\mathrm{CO}_{2}$ gas-phase abundances at radii $\leqslant 1 \mathrm{AU}$ due to a net inward transport from radii $\leqslant 10 \mathrm{AU}$. This leads to changes in the disk spectra for both models ACR and MIX: inside $2 \mathrm{AU}$, the gas and dust temperatures first decrease with increasing distance from the midplane (see Figure 1). This is due to the viscous heating near the midplane. Since $\mathrm{CO}_{2}$ is concentrated near the midplane and since it is abundant in model ACR (depleted in model MIX), the absorption feature at $15 \mu \mathrm{m}$ is enhanced (reduced).

Ammonia shows the largest variations between the different models. Compared to model A, model ACR and model MIX lead to a similar increase in the gas and ice abundances between 1 and $10 \mathrm{AU}$. The increase in ammonia is a result of a fundamental change in the oxygen chemistry. In model ACR and model MIX, atomic oxygen is reduced by orders of magnitude between 2 and $10 \mathrm{AU}$, with an increase in $\mathrm{O}_{2}$ and a decrease in $\mathrm{OH}$. The drastic decrease in atomic oxygen practically switches off the main destruction reaction of $\mathrm{NH}_{3}$,

$$
\mathrm{O}+\mathrm{NH}_{3} \longrightarrow \mathrm{OH}+\mathrm{NH}_{2} \text {. }
$$

The overabundant regions are located below the warm molecular layer in model ACR and reach slightly into it in model MIX (see also Figure 7). Hence, ammonia is found in absorption in the spectrum of model MIX due to the same reasons as $\mathrm{CO}_{2}$ in model ACR.

Another species of interest is methanol, whose gas-phase abundance is high in regions where the ammonia abundances are high in all the models. In model MIX, this implies an increase in methanol within 5 AU compared to model A. Viscous accretion increases its abundance between 2 and $5 \mathrm{AU}$ and decreases it further inside. As in model MIX, $\mathrm{CH}_{3} \mathrm{OH}$ is produced efficiently from $\mathrm{NH}_{3}$, however, its gas-phase formation and evaporation from dust grains at 5-10 AU are slower than its destruction and the accretion flow transports less material into the inner disk than is removed from it. Methanol is located below the transition region of the disk, even in model MIX, hence, the line opacities are so small that the absorption features are negligible in the spectra.

The abundance profile of acetylene can be explained as follows: in model MIX, its abundance is increased between 1 and $10 \mathrm{AU}$. Further inside, the diffusion leads to a depletion of $\mathrm{C}_{2} \mathrm{H}_{2}$. In model ACR, $\mathrm{C}_{2} \mathrm{H}_{2}$ is increased between 2 and $10 \mathrm{AU}$ and decreased further inside, similar to $\mathrm{CO}_{2}$. Since the overall abundance of $\mathrm{C}_{2} \mathrm{H}_{2}$ is low and since it is located below the 

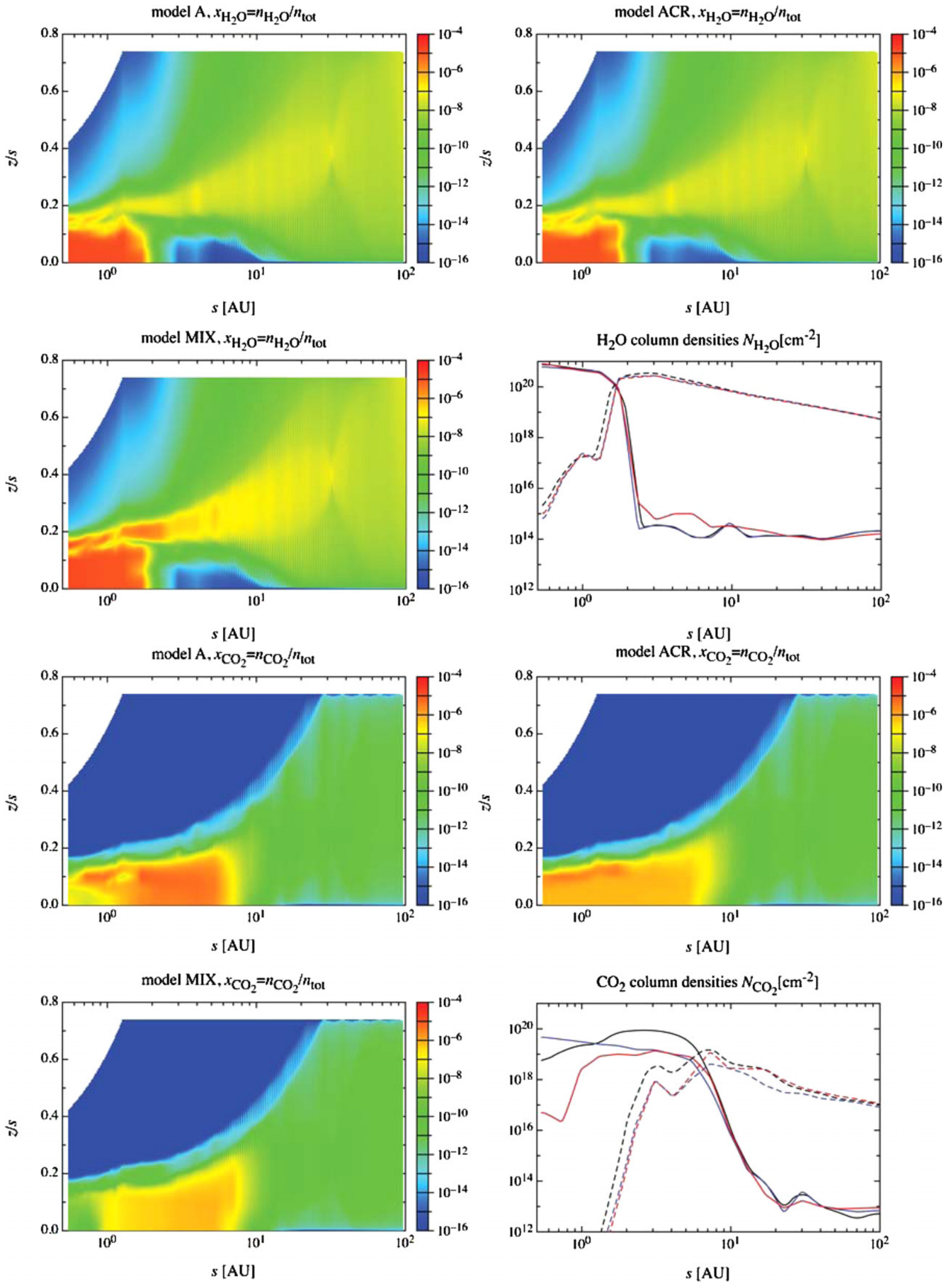

Figure 8. Two-dimensional fractional gas-phase abundances and column densities (solid: gas; dashed: ice) of $\mathrm{H}_{2} \mathrm{O}$ and $\mathrm{CO}_{2}$ for model A (black), model ACR (blue), and model MIX (red). Two-dimensional fractional gas-phase abundances and column densities (solid: gas; dashed: ice) of $\mathrm{NH}_{3}$ and $\mathrm{CH}_{3} \mathrm{OH}$ for model A (black), model ACR (blue), and model MIX (red). Two-dimensional fractional gas-phase abundances and column densities (solid: gas; dashed: ice) of $\mathrm{C}_{2} \mathrm{H}_{2}$ for model $\mathrm{A}$ (black), model ACR (blue), and model MIX (red).

(A color version of this figure is available in the online journal.) 

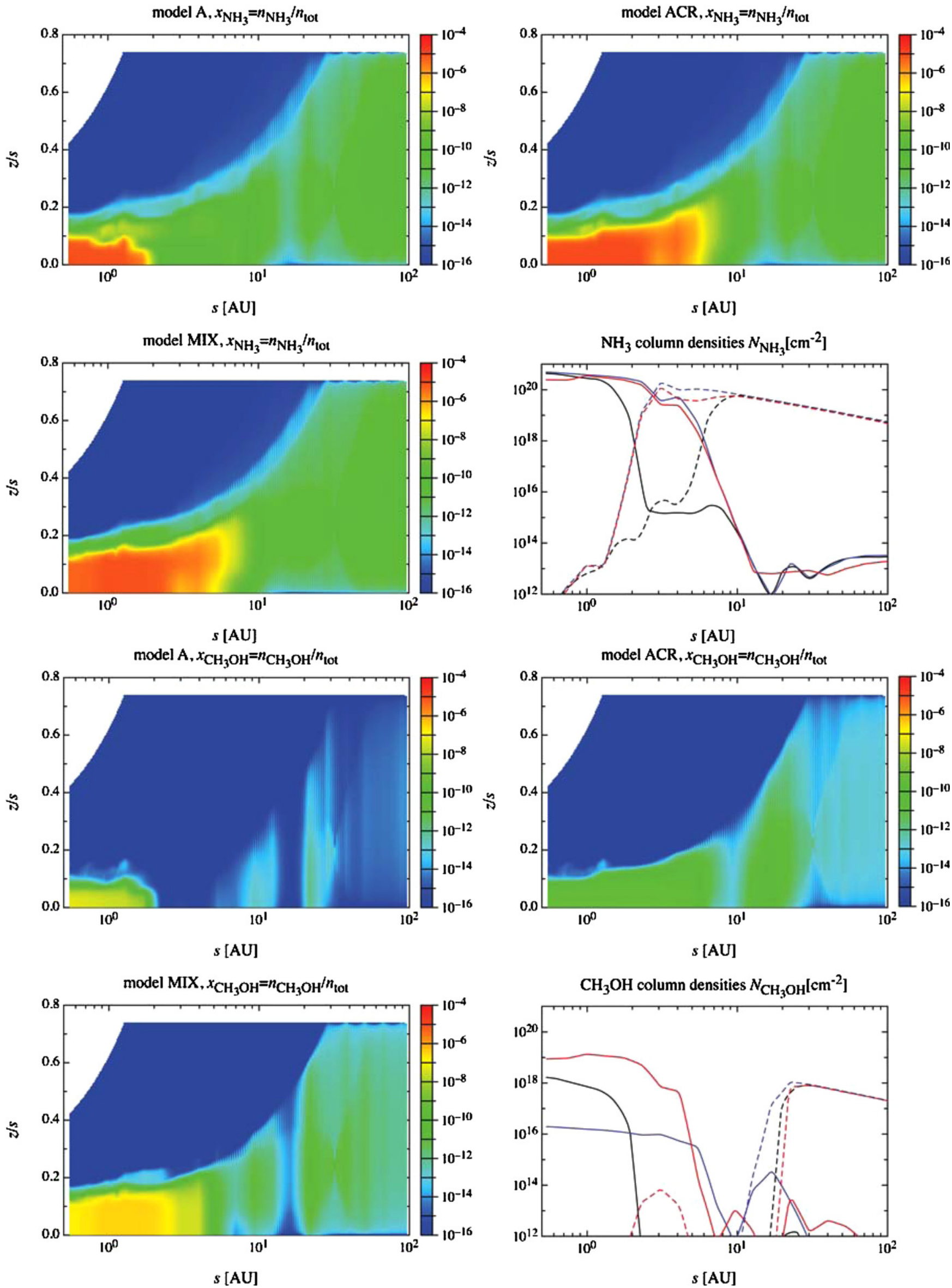

Figure 8. (Continued) 

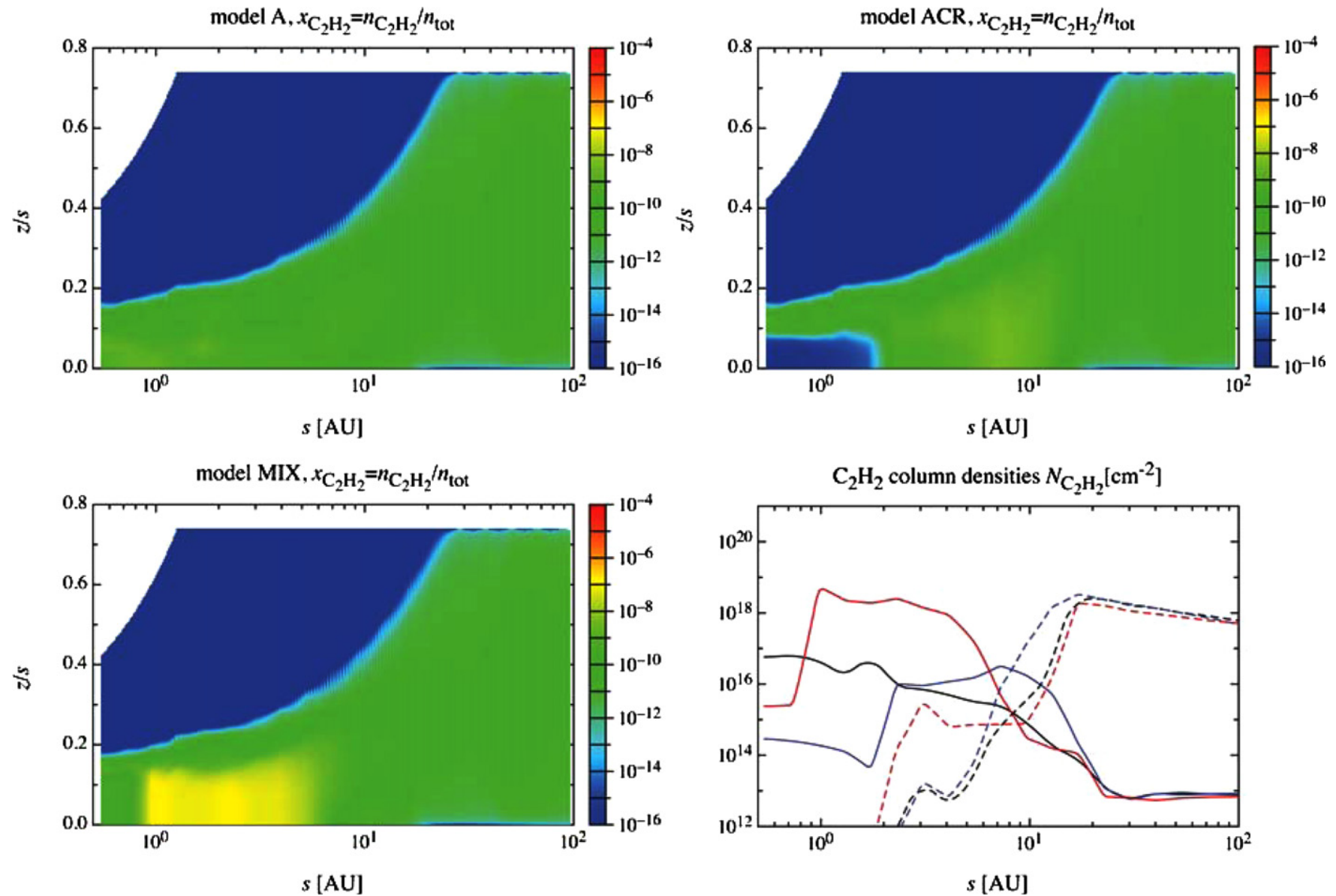

Figure 8. (Continued)

transition region, the absorption lines are negligibly small in the spectra of the disks in model A and model ACR. The stark increase in $\mathrm{C}_{2} \mathrm{H}_{2}$ in model MIX leads to a weak absorption feature seen at 13-13.5 $\mu \mathrm{m}$ (Figure 9).

\subsection{Theory versus Observations}

We refer to the observations of the protoplanetary disks of AA Tauri (Carr \& Najita 2008), DR Tauri, and AS 205 (Salyk et al. 2008). AA Tauri is a classical T Tauri star with mass $0.8 M_{\odot}$, accretion rate $10^{-8} M_{\odot} \mathrm{yr}^{-1}$, and inclination $75^{\circ}$ (Bouvier et al. 1999). Carr \& Najita (2008) observed the disk around AA Tauri with the Infrared Spectrograph (IRS) on the Spitzer Space Telescope over the wavelength range of 9.9-37.2 $\mu \mathrm{m}$. They found a rich spectrum of molecular emission lines dominated by rotational transitions of $\mathrm{H}_{2} \mathrm{O}$ and $\mathrm{OH}$. Between 10 and $15 \mu \mathrm{m}$, they detected rovibrational emission bands of $\mathrm{C}_{2} \mathrm{H}_{2}, \mathrm{HCN}$, and $\mathrm{CO}_{2}$, plus the atomic [Ne II] transition at $12.8 \mu \mathrm{m}$. Fundamental rovibrational emission bands of $\mathrm{CO}$ have been detected in Keck/NIRSPEC observations between 3 and $5 \mu \mathrm{m}$. Fitting model spectra to the observations, Carr \& Najita (2008) derive a high column density for these species and characteristic emission radii in the inner disk $(s<2.5 \mathrm{AU})$.

DR Tauri and AS 205, both classical T Tauri stars, have been observed with IRS on Spitzer and with Keck/NIRSPEC by Salyk et al. (2008). DR Tauri has a mass of $0.76 M_{\odot}$ and a variable accretion rate of $(0.3-79) \times 10^{-7} M_{\odot} \mathrm{yr}^{-1}$, much higher than in our model or that observed in AA Tauri. AS 205 is the primary component of a triple system with mass $1.2 M_{\odot}$ and accretion rate $7.2 \times 10^{-7} M_{\odot} \mathrm{yr}^{-1}$. The inclinations are $67^{\circ}$ for DR Tauri and $47^{\circ}$ for AS $205 \mathrm{~A}$, respectively. Both systems show a large number of transitional emission lines from water and require high temperatures and column densities to explain this emission, similar to AA Tauri. Further, $\mathrm{CO}, \mathrm{OH}$, and $\mathrm{CO}_{2}$ are required to fit the observed spectra in the near- to midinfrared. In the following, we discuss how our models relate to, and compare with, these observational results.

The calculated spectra for our models show emission and absorption features different from the observed spectra (Figure 9). First, the molecular line emission in our models is dominated by rotational transitions of $\mathrm{OH}$. Weak emission from $\mathrm{H}_{2} \mathrm{O}$ is detected in model MIX. In model ACR and in model A, water is found mainly in absorption. Model WND leads to results similar to model A due to the limitations of our disk model. These models make use of the simple $\mathrm{H}_{2}$ formation rates on grains, Equation (2). Model B, which accounts for the enhanced $\mathrm{H}_{2}$ formation rates on grains, Equation (4), leads to huge emission lines from $\mathrm{OH}$ and $\mathrm{H}_{2} \mathrm{O}$. The near-infrared emission bands of $\mathrm{CO}$ at $5 \mu \mathrm{m}$ are reproduced in model $\mathrm{A}$, model MIX, and also in model ACR.

The modeled line intensities depend strongly on the gas temperature and molecular abundances profiles, i.e., the detailed thermal balance and the chemistry. It is therefore more favorable to compare the molecular column densities from our model calculations to the observed ones. In the inner disk, the observed column densities and abundance ratios are derived from the photodestruction layer and the warm molecular layer, i.e., from the disk surface down to an optical depth of about unity. 

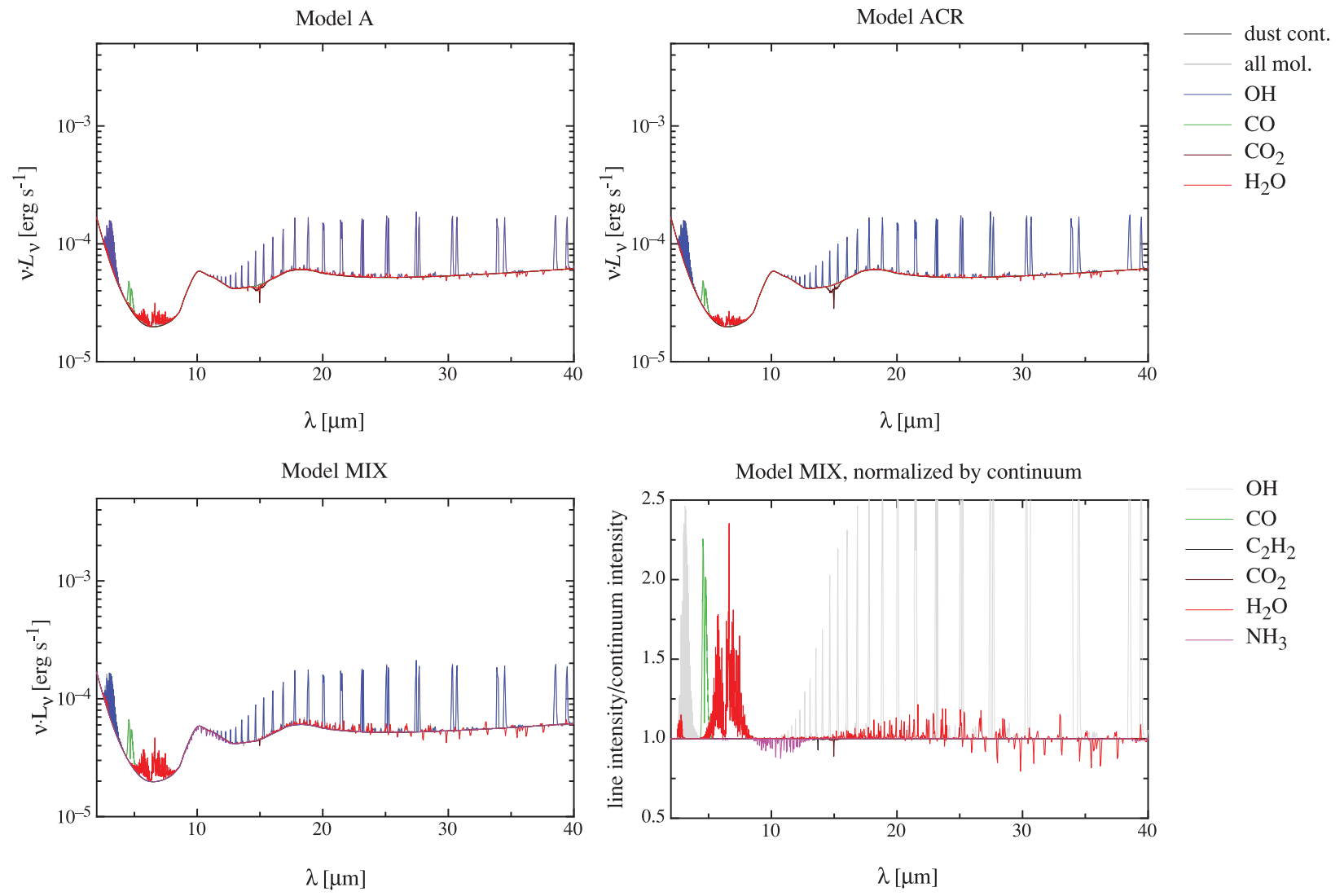

Figure 9. LTE line spectra for model A, model ACR, and model MIX for an observer at inclination $i=0$. For model MIX, we also show the spectrum normalized by the continuum flux in the lower right panel (for a better illustration, the color coding in the normalized spectrum is different from the other spectra and listed in the lower right legend).

(A color version of this figure is available in the online journal.)

The total molecular column densities in our model are mainly determined by the dense midplane layers and are therefore larger by orders of magnitude and not comparable to the observed column densities. Thus, we calculate the column densities of the observed species from the disk surface down to a continuum optical depth of unity at each wavelength and compare them to the observations (Figure 10). At $s=1.3 \mathrm{AU}$ and wavelengths $\lambda=\{5,10,20,40\} \mu \mathrm{m}$, the disk becomes optically thick at $z / s=\{0.14,0.15,0.14,0.11\}$, respectively. For the unusually strong $\mathrm{OH}$ lines, a continuum optical depth of unity corresponds to a maximum optical depth of $\tau \sim 20$ (see the discussion below for large discrepancy between the models and the observations of $\mathrm{OH}$ lines). For all other molecules, the maximum optical depth of the lines is $\tau \sim 2$.

If we compare the $\mathrm{H}_{2} \mathrm{O}$ and $\mathrm{CO}$ column densities of the upper layers at disk radius $1.3 \mathrm{AU}$, we find an abundance ratio of 0.02 for model A and model ACR, while model MIX shows an abundance ratio of 0.18. Carr \& Najita (2008) derive an abundance ratio of $\mathrm{H}_{2} \mathrm{O}$ to $\mathrm{CO}$ of 1.3, while Salyk et al. (2008) find water to be less abundant than carbon monoxide with an abundance ratio for $\mathrm{H}_{2} \mathrm{O}$ to $\mathrm{CO}$ of 0.1 for both systems. Note that Carr \& Najita (2008) calculate the abundance ratio for different radii of the $\mathrm{H}_{2} \mathrm{O}$ and $\mathrm{CO}$ column densities (2.1 $\mathrm{AU}$ and $0.7 \mathrm{AU}$ ), while Salyk et al. (2008) obtain their results for the same radius (3 AU). It is important to note that the observed systems differ from our model in their central objects, disk masses, inclination angles, and accretion rates, with AA Tauri being the closest match. Further, the derived column densities in Carr \& Najita (2008) and Salyk et al. (2008) contain considerable uncertainties in their values and their characteristic radii.
The resulting column densities of the upper disk layers are displayed in Figure 10 for model A and for model MIX only, since it leads to the largest changes in the vertical distribution of the chemical species. In model MIX, the $\mathrm{H}_{2} \mathrm{O}$ to $\mathrm{CO}$ abundance ratio is of the order of 0.2 inside the snow line, while in model $\mathrm{A}$, it is reduced by at least a factor of 10 . Model MIX reproduces the $\mathrm{H}_{2} \mathrm{O}$ abundance in AA Tauri at a radius of 2.1 AU. In the case of DR Tauri and AS 205, the central star is more luminous than in AA Tauri and in our model, hence, the snow line is located further outward and gas-phase $\mathrm{H}_{2} \mathrm{O}$ remains abundant with derived column densities of the same order as in our model or in AA Tauri at radii of $2 \mathrm{AU}$. The modeled $\mathrm{CO}$ abundances agree with the observations for both models. Both models fail to reproduce the high $\mathrm{OH}$ abundances, although the calculated line emission is much stronger than that of the observations. This adds further support to the call for non-LTE spectral calculations and gas temperature profile calculations accounting for dustgrain settling and grain growth (cf. Section 3.1). Since the binding energy of $\mathrm{OH}$ on dust grains is low and considering it is robust against $\mathrm{UV}$ radiation, efficient turbulent mixing can increase the abundances in the upper layers of the disk (cf. Figure 10).

$\mathrm{CO}_{2}$ is found in absorption instead of in emission in model $\mathrm{A}$, model ACR, and model MIX. The absorption is strongest in model ACR and weakest in model MIX. Contrary to this, the observations detect $\mathrm{CO}_{2}$ in emission at a characteristic radius of $1.2 \mathrm{AU}$ in AA Tauri. It is also detected in emission toward DR Tauri and AS 205 (with large uncertainties on the column densities and the emission radii). The column densities derived from the observations of AA Tauri are lower than in model A 
Column densities of the upper disk layers
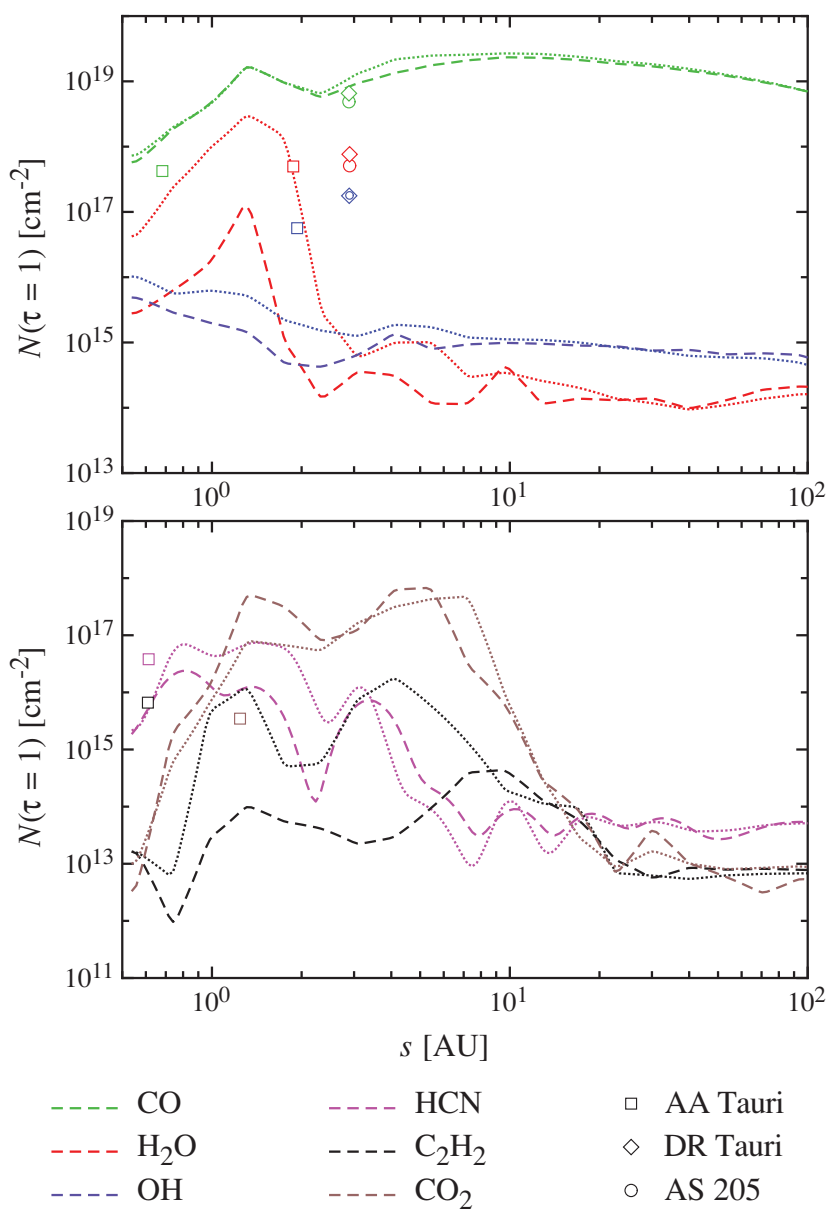

Figure 10. Column densities of the gas-phase species in the upper layers in model A (dashed) and model MIX (dotted). The derived column densities for AA Tauri, DR Tauri, and AS 205 are indicated at their characteristic radii (Carr \& Najita 2008; Salyk et al. 2008).

(A color version of this figure is available in the online journal.)

and model MIX, although the latter one gives a better fit. Since $\mathrm{CO}_{2}$ is destroyed quickly in the upper layers of the disk, stronger mixing will lead to lower $\mathrm{CO}_{2}$ abundances.

$\mathrm{HCN}$ is detected in weak emission in AA Tauri and a number of other sources (see, for example, Pontoppidan et al. 2010), but not detected in our modeled spectra. The column density in AA Tauri is slightly higher than in our models, and model MIX again gives a better fit. Turbulent mixing increases the column density in the upper layers, although the total column density is reduced. Stronger mixing will help to improve the fit of the model calculations to the observations.

In the case of $\mathrm{C}_{2} \mathrm{H}_{2}$, the characteristic radius in AA Tauri could not be determined and was set to that derived for $\mathrm{HCN}$. Assuming that the $\mathrm{C}_{2} \mathrm{H}_{2}$ emission is located at larger radii (e.g., $1 \mathrm{AU}$ instead of $0.6 \mathrm{AU})$, model MIX fits the observed column density, while model A predicts much lower values. Adversely, the spectrum of model MIX shows $\mathrm{C}_{2} \mathrm{H}_{2}$ in weak absorption instead of emission. From the previous discussion in Sections 3.2 and 3.3, we can assume that stronger turbulence will transport $\mathrm{C}_{2} \mathrm{H}_{2}$ higher up in the disk, leading to emission instead of absorption signatures.

The abundances of $\mathrm{NH}_{3}$ are enhanced in model MIX, leading to absorption signatures in the spectrum between 8 and $13 \mu \mathrm{m}$.
To date, there has been no published detection of ammonia from protoplanetary disks, but $\mathrm{NH}_{3}$ has been found in absorption toward the hot core associated with the massive protostar NGC 7538 IRS 1 (Knez et al. 2009), using the Texas Echelon Cross Echelle Spectrograph (TEXES). Observing $\mathrm{NH}_{3}$ emission from disks with high resolution and high sensitivity will allow us to draw important conclusions on the efficiency of turbulent mixing in protoplanetary disks.

Taking into account the model uncertainties, e.g., the $\mathrm{H}_{2}$ formation rates on grains, the gas temperature profiles (which are related to the dust model adopted, e.g., to the dust growth and settling), as well as the uncertainties in deriving molecular column densities and characteristic radii from the observations, further investigations are needed to strengthen the significance of our results.

In addition, the observed objects have inclination angles of $67^{\circ}$ for DR Tauri and $47^{\circ}$ for AS 205A, while our theoretical spectra are calculated for a disk seen face-on. This affects the observed line emission and the derived column densities, since the chemical composition is different along the line of sight. Robust conclusions therefore require spectral calculations accounting for the different inclination angles.

\section{CONCLUSION}

We studied the chemical evolution of a steady protoplanetary disk, investigating different models for the formation rate of $\mathrm{H}_{2}$ on dust grains and the effects of including viscous accretion, turbulent mixing, and disk winds. Using our chemical results, we calculated the emergent near- and mid-infrared spectrum from the disk, including contributions from the dust continuum and molecular line emission.

Our results show that the abundances of water and the hydroxyl radical are very sensitive to the $\mathrm{H}_{2}$ formation rates on grains, with the resulting spectra differing considerably. The enhanced $\mathrm{H}_{2}$ formation rates of Cazaux \& Tielens (2002) lead to a drastic increase in the gas-phase abundances of water and $\mathrm{OH}$ in the upper disk layers and to strong emission lines. We concluded that dust grain settlement toward the midplane and non-LTE calculations of the molecular line emission are required to obtain meaningful results for models using these enhanced $\mathrm{H}_{2}$ formation rates.

We investigated the effects of physical mass transport phenomena in the radial direction by viscous accretion and in the vertical direction by diffusive turbulent mixing and disk winds. Due to the steady state assumption of the underlying protoplanetary disk model, the wind velocities are limited to impede the evaporation of the disk over its lifetime and consequently, the effects of the disk winds on the chemistry are small. On the contrary, vertical diffusive mixing has a considerable impact on the disk chemistry and increases the abundance of many observed species in the warm molecular layer of the disk. Species which are produced efficiently in a particular layer and which are stable in lower (or upper) layers can experience a significant enhancement due to the diffusive motion. Including the radial accretion flow in the chemical network calculation leads to large changes in the chemical composition of the disk, particularly in the cold and dense midplane layer. The effect of the accretion flow on a particular species depends on the accretion timescale and the formation and destruction timescales of that species. For example, inside $10 \mathrm{AU}$, the gasphase water abundances are not overly affected by the accretion flow, while ammonia is greatly enhanced and methanol greatly depleted. 

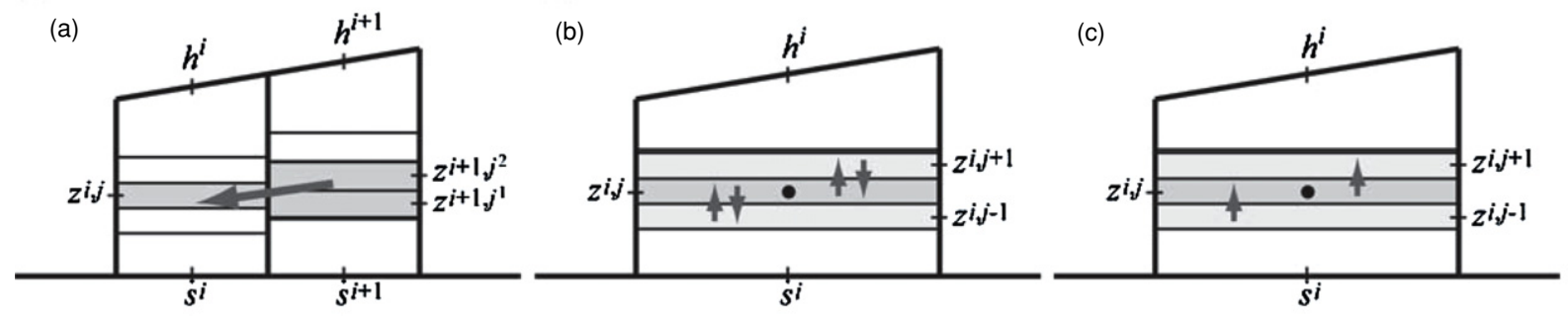

Figure 11. Schematic view of (a) accretion, (b) mixing, and (c) wind modeling in our model.

Since the accretion flow affects mainly the midplane layers, the infrared molecular line emission is basically unchanged. Contrarily, turbulent mixing alters the chemical composition of the warm molecular layer, where the molecular line emission is generated. We thus contrasted two models with and without turbulent mixing to observations of protoplanetary disks around classical T Tauri stars. We compared the modeled disk spectra and column densities of the upper layers of the disk to the observed spectra and the derived column densities.

In summary, a model with turbulent mixing reproduces the observations of AA Tauri, DR Tauri, and AS 205 better than a model without mixing, although the discussion of the column densities in the upper layers and of the spectra suggests a more efficient vertical mass transport mechanism. In the models considered here, turbulent mixing provides an efficient way of transporting material from the midplane into the transition layer. Due to the constant mixing velocity in each vertical column, the turbulent timescales become longer than the chemical timescales at larger distances from the midplane. But these are the layers where the disk winds studied by Suzuki \& Inutsuka (2009) become most effective (the transition layer coincides roughly with 1.5 disk scale heights) and drive large-scale channel flows. Hence, a combination of turbulence in the cold midplane layers and disk winds in the transition layer, both generated by the magneto-rotational instability, might account for the observations better than turbulent mixing or disk winds alone. In the latter case, the steady state assumption would have to be abandoned to allow for stronger disk winds.

An interesting aspect for future studies will be the combination of radial and vertical transport processes. In this work, we showed that the radial accretion flow alters predominantly the midplane abundances, while turbulent mixing affects the midplane and warm molecular layers. We therefore expect that a combination of radial and vertical motion will have a strong impact on the chemical abundances in the molecular layer, which will be reflected in the emission line spectrum.

The authors thank Dr. Inutsuka and Dr. Suzuki from Nagoya University for providing the data of their MHD simulations and for useful discussions. D.H. and H.N. were supported by the Grant-in-Aid for the Global COE Program "The Next Generation of Physics, Spun from Universality and Emergence" from the Ministry of Education, Culture, Sports, Science, and Technology (MEXT) of Japan. D.H. received further support from the Kyoto University Startup Grants, and H.N. from the Grant-in-Aid for Scientific Research 2174 0137, MEXT. C.W. acknowledges DEL for a studentship and JSPS for the award of a short-term fellowship to conduct research in Japan. Astrophysics at QUB is supported by a grant from the STFC.

The numerical calculations were carried out on Altix 3700 BX2 at the Yukawa Institute for Theoretical Physics, Kyoto University, and on the Cray XT4C at the Center for Compu- tational Astrophysics, National Astronomical Observatory of Japan.

\section{APPENDIX}

\section{PHYSICOCHEMICAL INTERACTIONS-NUMERICS}

Modeling the physical mass transport phenomena in combination with calculating the chemical evolution is the key aspect of this study. Here, we discuss in detail the numerical techniques used to compute the chemistry accounting for transport mechanisms in relation to the grid of our protoplanetary disk model.

The general expression for the rate of change in the abundance of gas-phase species $k$ in grid cell $(i, j)$ at radius $s^{i}$ and vertical distance $z^{j}$ from the midplane is given by

$$
\dot{n}_{k}^{i, j}=\frac{\Delta n_{k}^{i, j}}{\Delta t}=P_{k}^{i, j}-L_{k}^{i, j}-\dot{n}_{k \text { ice }}^{i, j}+\dot{n}_{k, \text { acc } / \mathrm{mix} / \mathrm{wind}}^{i, j},
$$

where $P_{k}$ and $L_{k}$ denote the production and loss terms, respectively, due to gas-phase chemical reactions, $\dot{n}_{k \text { ice }}$ the adsorption/ desorption rate ( $>0$ for net freeze out, $<0$ for net evaporation), and $\dot{n}_{k \text {, acc/mix } / \text { wind }}$ the rates arising from mass transport due to accretion, mixing, or disk winds. In the case of molecular hydrogen, an additional (positive) term $\dot{n}_{\mathrm{H}_{2}}$ is added to the right-hand side to account for the $\mathrm{H}_{2}$ formation on grains. Accordingly, in the case of atomic hydrogen, an additional term $-2 \dot{n}_{\mathrm{H}_{2}}$ is required.

\section{A.1. Radial Transport by Viscous Accretion}

We assume that inward accretion occurs along constant streamlines $z / h$. (see Figure 11(a) for an illustration of the coupling between the grid cells at different radii). The net accretion rate along constant streamlines is given in Equation (15), where the differentiation with respect to $l$ needs to be translated into a differentiation with respect to $s$. Accordingly, on the discrete grid of the disk model, the net accretion rate for cell $(i, j)$ can be expressed as

$$
\dot{n}_{k, \text { acc }}^{i, j}=\frac{\bar{v}_{s}^{i, i+1}}{\Delta s^{i}}\left\{-n_{k}^{i, j}+\frac{\tilde{\rho}^{i+1, j^{1}, j^{2}}}{\rho^{i, j}} \tilde{n}_{k}^{i+1, j^{1}, j^{2}}\right\},
$$

where $\tilde{\rho}$ and $\tilde{n}$ represent the average mass and number density of the outer grid cells, respectively, weighed by their relative mass transfer into cell $(i, j)$. The accretion velocity $\bar{v}_{s}^{i, i+1}$ is evaluated at the interface between the grid cells $i$ and $i+1$ and is introduced to improve the accuracy of the finite calculation. The additional factor $\tilde{\rho}^{i+1, j^{1}, j^{2}} / \rho^{i, j}$ arises from the requirement of mass conservation. In the context of a steady disk model, all variables except $n_{k}^{i, j}$ and $\tilde{n}_{k}^{i+1, j^{1}, j^{2}}$ are constant with time. 


\section{A.2. Vertical Transport by Turbulent Mixing}

Figure 11(b) demonstrates the grid-cell mass transfer by turbulent mixing in the diffusion-type model. Starting from Equation (18), we replace the differentials by finite differences over the vertical extent $\Delta z$ of the grid cells. Additionally, we introduce mean densities $\bar{\rho}$ and velocities $\bar{v}$ at the interface of the grid cells as before. The exchange rates of species $k$ between grid cell $(i, j)$ and the adjacent grid cells $(i, j-1)$ and $(i, j+1)$ then become

$$
\begin{aligned}
\dot{n}_{k, \text { mix }}^{i, j}= & \frac{\bar{\rho}^{i, j-1, j}}{\rho^{i, j-1}} \cdot \frac{\bar{v}_{z}^{i, j-1, j}}{\Delta z^{i, j}} \cdot\left(n_{k}^{i, j-1}-\frac{\rho^{i, j-1}}{\rho^{i, j}} n_{k}^{i, j}\right) \\
& +\frac{\bar{\rho}^{i, j, j+1}}{\rho^{i, j}} \cdot \frac{\bar{v}_{z}^{i, j, j+1}}{\Delta z^{i, j}} \cdot\left(\frac{\rho^{i, j}}{\rho^{i, j+1}} n_{k}^{i, j+1}-n_{k}^{i, j}\right) .
\end{aligned}
$$

\section{A.3. Vertical Transport by Disk Winds}

The model of the upward disk wind is shown in Figure 11(c). In the finite case, the differential equation for the wind rate (Equation (20)) of grid cell $(i, j)$ translates into

$$
\dot{n}_{k, \text { wind }}^{i, j}=\frac{v_{z}^{i, j-1, j}}{\Delta z^{i, j}} \cdot n_{k}^{i, j-1}-\frac{v_{z}^{i, j, j+1}}{\Delta z^{i, j}} n_{k}^{i, j} .
$$

In both the wind and the mixing scenarios, the only variable changing with time is $n_{k}$.

\section{REFERENCES}

Aikawa, Y., Miyama, S. M., Nakano, T., \& Umebayashi, T. 1996, ApJ, 467, 684

Aikawa, Y., \& Nomura, H. 2006, ApJ, 642, 1152

Aikawa, Y., Umebayashi, T., Nakano, T., \& Miyama, S. M. 1999, ApJ, 519, 705 Aikawa, Y., van Zadelhoff, G. J., van Dishoeck, E. F., \& Herbst, E. 2002, A\&A, 386,622

Armitage, P. J. 2007, ApJ, 665, 1381

Beckwith, S. V. W., Sargent, A. I., Chini, R. S., \& Guesten, R. 1990, AJ, 99, 924

Blandford, R. D., \& Payne, D. G. 1982, MNRAS, 199, 883

Bouvier, J., et al. 1999, A\&A, 349, 619

Brittain, S. D., Najita, J. R., \& Carr, J. S. 2009, ApJ, 702, 85

Brown, W. A., \& Bolina, A. S. 2007, MNRAS, 374, 1006

Carmona, A. 2010, Earth Moon Planets, 106, 71

Carmona, A., van den Ancker, M. E., Henning, Th., Goto, M., Fedele, D., \& Stecklum, B. 2007, A\&A, 476, 853

Carr, J. S. 2007, in IAU Symp. 243, Star-Disk Interaction in Young Stars, ed. J. Bouvier \& I. Appenzeller (Cambridge: Cambridge Univ. Press), 135

Carr, J. S., \& Najita, J. R. 2008, Science, 319, 1504

Cazaux, S., \& Tielens, A. G. G. M. 2002, ApJ, 575, L29

Charnley, S. B. 1997, ApJ, 481, 396

Courant, R., Friedrichs, K., \& Lewy, H. 1928, Math. Ann., 100, 32

Dominik, C., Blum, J., Cuzzi, J. N., \& Wurm, G. 2007, in Protostars and Planets V, ed. B. Reipurth, D. Jewitt, \& K. Keil (Tucson, AZ: Univ. Arizona Press), 783

Draine, B. T. 1978, ApJS, 36, 595

Duley, W. W., \& Williams, D. A. 1984, Interstellar Chemistry (London: Academic)

Dullemond, C. P., \& Turolla, R. 2000, A\&A, 360, 1187

Dullemond, C. P., van Zadelhoff, G. J., \& Natta, A. 2002, A\&A, 389, 464

Dutrey, A., Guilloteau, S., \& Ho, P. 2007, in Protostars and Planets V, ed. B. Reipurth, D. Jewitt, \& K. Keil (Tucson, AZ: Univ. Arizona Press), 495

Fogel, J. K. J., Bethell, T. J., Bergin, E. A., Calvet, N., \& Semenov, D. 2011, ApJ, 726,29

Geers, V. C., et al. 2006, A\&A, 459, 545

Gibb, E. L., Van Brunt, K. A., Brittain, S. D., \& Rettig, T. W. 2007, ApJ, 660, 1572

Glassgold, A. E., Meijerink, R., \& Najita, J. R. 2009, ApJ, 701, 142

Glassgold, A. E., Najita, J., \& Igea, J. 2004, ApJ, 615, 972

Gorti, U., \& Hollenbach, D. 2004, ApJ, 613, 424
Gould, R. J., \& Salpeter, E. E. 1963, ApJ, 138, 393

Habart, E., Natta, A., Testi, L., \& Carbillet, M. 2006, A\&A, 449, 106

Haisch, K. E., Jr., Barsony, M., Ressler, M. E., \& Greene, T. P. 2006, AJ, 132, 2675

Haisch, K. E., Jr., Lada, E. A., \& Lada, C. J. 2001, ApJ, 553, L153

Hasegawa, T. I., \& Herbst, E. 1993, MNRAS, 261, 83

Hatchell, J., Thompson, M. A., Millar, T. J., \& MacDonald, G. H. 1998, A\&A, 338,713

Hogerheijde, M., \& van der Tak, F. 2000, A\&A, 362, 697

Hollenbach, D., \& McKee, C. F. 1979, ApJS, 41, 555

Hollenbach, D., \& Salpeter, E. E. 1971, ApJ, 163, 155

Ilgner, M., Henning, Th., Markwick, A. J., \& Millar, T. J. 2004, A\&A, 415, 643

Jonkheid, B., Faas, F. G. A., van Zadelhoff, G.-J., \& van Dishoeck, E. F. 2004, A\&A, 428, 511

Kamp, I., \& Dullemond, C. P. 2004, ApJ, 615, 991

Knez, C., Lacy, J. H., Evans, N. J., van Dishoeck, E. F., \& Richter, M. J. 2009, ApJ, 696, 471

Lada, C. J., \& Wilking, B. A. 1984, ApJ, 287, 610

Lahuis, F., et al. 2006, ApJ, 636, L145

Markwick, A. J., Ilgner, M., Millar, T. J., \& Henning, Th. 2002, A\&A, 385, 632

Meijerink, R., Pontoppidan, K. M., Blake, G. A., Poelman, D. R., \& Dullemond, C. P. 2009, ApJ, 704, 1471

Millar, T. J., MacDonald, G. H., \& Gibb, A. G. 1997, A\&A, 325, 1163

Najita, J. R., Carr, J. S., Glassgold, A. E., \& Valenti, J. A. 2007, in Protostars and Planets V, ed. B. Reipurth, D. Jewitt, \& K. Keil (Tucson, AZ: Univ. Arizona Press), 507

Natta, A., Testi, L., Calvet, N., Henning, Th., Waters, R., \& Wilner, D. 2007, in Protostars and Planets V, ed. B. Reipurth, D. Jewitt, \& K. Keil (Tucson, AZ: Univ. Arizona Press), 767

Nomura, H. 2002, ApJ, 567, 587

Nomura, H., Aikawa, Y., Nakagawa, Y., \& Millar, T. J. 2009, A\&A, 495, 183

Nomura, H., Aikawa, Y., Tsujimoto, M., Nakagawa, Y., \& Millar, T. J. 2007, ApJ, 661,334

Nomura, H., \& Millar, T. J. 2005, A\&A, 438, 923

Ossenkopf, V., \& Henning, Th. 1994, A\&A, 291, 943

Pontoppidan, K. M., Blake, G. A., van Dishoeck, E. F., Smette, A., Ireland, M. J., \& Brown, J. 2008, ApJ, 684, 1323

Pontoppidan, K. M., Salyk, C., Blake, G. A., Meijerink, R., Carr, J. S., \& Najita, J. R. 2010, ApJ, 720, 887

Pringle, J. E. 1981, ARA\&A, 19, 137

Qi, C., Wilner, D. J., Calvet, N., Bourke, T. L., Blake, G. A., Hogerheijde, M. R., Ho, P. T. P., \& Bergin, E. 2006, ApJ, 636, L157

Rodgers, S. D., \& Charnley, S. B. 2001, ApJ, 546, 324

Rothman, L. S., et al. 2005, J. Quant. Spectrosc. Radiat. Transfer, 96, 139

Salyk, C., Pontoppidan, K. M., Blake, G. A., Lahuis, F., van Dishoeck, E. F., \& Evans, N. J. 2008, ApJ, 676, L49

Sandford, S. A., \& Allamondola, L. J. 1988, Icarus, 76, 201

Sandford, S. A., \& Allamondola, L. J. 1990, Icarus, 87, 188

Sandford, S. A., \& Allamondola, L. J. 1993, ApJ, 417, 815

Semenov, D., Wiebe, D., \& Henning, Th. 2006, ApJ, 647, L57

Shakura, N. I., \& Sunyaev, R. A. 1973, A\&A, 24, 337

Shu, F., Johnstone, D., \& Hollenbach, D. 1994, Icarus, 106, 92

Shu, F., Najita, J., Ostriker, E., Wilkin, F., Ruden, S., \& Lizano, S. 1993, ApJ, 429, 781

Suzuki, T. K., \& Inutsuka, S. 2009, ApJ, 691, L49

van der Plas, G., van den Ancker, M. E., Acke, B., Carmona, A., Dominik, C., Fedele, D., \& Waters, L. B. F. M. 2009, A\&A, 500, 1137

van Dishoeck, E. F., \& Black, J. H. 1982, ApJ, 258, 533

Walsh, C., Millar, T. J., \& Nomura, H. 2010, ApJ, 722, 1607

Watson, A. M., Stapefeldt, K. R., Wood, K., \& Ménard, F. 2007, in Protostar and Planets V, ed. B. Reipurth, D. Jewitt, \& K. Keil (Tucson, AZ: Univ. Arizona Press), 523

Weingartner, J. C., \& Draine, B. T. 2001a, ApJ, 548, 296

Weingartner, J. C., \& Draine, B. T. 2001b, ApJS, 134, 263

Westley, M. S., Baragiola, R. A., Johnson, R. E., \& Baratta, G. A 1995, Planet. Space Sci., 43, 1311

Willacy, K. 2007, ApJ, 660, 441

Willacy, K., Langer, W., Allen, M., \& Bryden, G. 2006, ApJ, 644, 1202

Woitke, P., Kamp, I., \& Thi, W.-F. 2009a, A\&A, 501, 383

Woitke, P., Thi, W.-F., Kamp, I., \& Hogerheijde, M. R. 2009b, A\&A, 501, L5

Wolk, S. J., Harnden, F. R., Flaccomio, E., Micela, G., Favata, F., Shang, H., \& Feigelson, E. D. 2005, ApJS, 160, 423

Woodall, J., Agúndez, M., Markwick-Kemper, A. J., \& Millar, T. J. 2007, A\&A, 466, 1197

Woods, P. M., \& Willacy, K. 2009, ApJ, 693, 1360

Yamamoto, T., Nakagawa, N., \& Fukui, Y. 1983, A\&A, 122, 171 